\title{
ENCODING OF TARGET RANGE AND ITS REPRESENTATION IN THE AUDITORY CORTEX OF THE MUSTACHED BAT ${ }^{1}$
}

\author{
WILLIAM E. O'NEILL ${ }^{2}$ AND NOBUO SUGA
}

\author{
Department of Biology, Washington University, St. Louis, Missouri 63130
}

Received August 3, 1981; Revised September 28, 1981; Accepted September 29, 1981

\begin{abstract}
The time course of acoustic events is a critical element for the recognition of biologically rneaningful sounds. Echolocating bats analyze the time intervals between their emitted biosonar pulses and the echoes returning from objects to assess target distance (range). In this study, we have explored the auditory cortex of the mustached bat, Pteronotus parnellii rubiginosus, using pairs of acoustic stimuli mimicking the multiharmonic biosonar signals (pulses) used by this species and their echoes.

A discrete field of auditory cortex dorsorostral to the tonotopically organized primary field contains neurons which are insensitive to pure tone, frequency-modulated (FM), or noise stimuli presented singly. Rather, they respond strongly to pairs of stimuli, specifically, the fundamental FM component of the pulse paired with an FM component of one of the higher harmonics of the echo. We call these neurons $\mathrm{FM}_{1}-\mathrm{FM}_{n}$ facilitation neurons. There are three separate longitudinal clusters in this cortical area containing $\mathrm{FM}_{1}-\mathrm{FM}_{2}, \mathrm{FM}_{1}-\mathrm{FM}_{3}$, and $\mathrm{FM}_{1}-\mathrm{FM}_{4}$ neurons, respectively. Moreover, $\mathrm{FM}_{1}-\mathrm{FM}_{n}$ neurons are specifically sensitive to the time delay between the two FM components, i.e., the time delay of the echo from the pulse. Thus, they can decode target range.

Two types of delay-sensitive neurons were found. Tracking neurons, whose response to echo delay varied according to repetition rate and stimulus duration, were found rarely. Delay-tuned neurons, which were tuned to specific time delays (best delays) of the echo from the pulse, were much more evident. Both types of neurons are organized into columns with similar best delays, and the best delay of delay-tuned neurons was found to increase systematically along the cortical surface in the rostrocaudal direction. This area, therefore, contains a neural representation of target range along this best delay axis. Such an axis exists in each of the clusters of $\mathrm{FM}_{1}-\mathrm{FM}_{2}, \mathrm{FM}_{1}-\mathrm{FM}_{3}$, and $\mathrm{FM}_{1}-\mathrm{FM}_{4}$ neurons. This is a new type of cortical organization which is not tonotopic but which represents an important acoustic cue related to the time course of acoustic events.
\end{abstract}

Acoustic signals can be described in terms of physical parameters, such as frequency, amplitude, and time. In mammals, vocal signals are composed typically of discrete utterances called phonemes, which are ordered in

This work was supported by National Science Foundation Grants BMS 75-17077 and BNS 78-12987 to N. S. W. E. O. wishes to thank the faculty of the Sensory Physiology group for postdoctoral training received under the auspices of the Sensory Physiology Training Grant (National Institute of Neurological and Communicative Disorders and Stroke Grant 1-T32-NS07057-01) administered through the Department of Physiology and Biophysics, Washington University Medical School. We are indebted to James Jaeger for technical assistance, Peter Wasserbach for the design and construction of the harmonic generators used in these experiments, and Nancy Dintruff and Mary Lee Stein of the University of Rochester for preparing the manuscript.

${ }^{2}$ To whom correspondence should be addressed at his present address: Center for Brain Research, University of Rochester Medical School, Rochester, NY 14642. time to convey information. Although the temporal patterns within phonemes and their sequences in the acoustic stream carry much of the total information in vocalizations, research in mammalian audition has focused mainly on the analysis and representation of frequency in the auditory system. That the cochlea is primarily a frequency analyzer (von Békésy, 1960) probably accounts for much of this emphasis. Another factor in favor of this bias is the relative ease of generation and the physical simplicity of pure tone stimuli for investigating the properties of the auditory system.

A major result of this endeavor has been to show that central auditory organization is dominated by the recapitulation of the morphology of the sensory epithelium, i.e., the organ of Corti. Mapping of peripheral organization onto the central nervous system is a property common to the visual and somatosensory systems as well. For audition, the representation of frequency along the 
cochlear partition is repeated throughout the central auditory system by tonotopic representation. Despite its pervasiveness, the functional significance of tonotopic representation in the cerebral cortex remains obscure, except perhaps for certain species of bats (Suga and Jen, 1976; Ostwald, 1980).

Since auditory information can be carried by several different parameters in sounds, the auditory cortex is likely to be involved in analysis of other acoustic features besides frequency. In this paper, we address directly the representation of temporal information in the central auditory system of the mustached bat, Pteronotus parnellii rubiginosus. By comparing these results with a previous investigation of frequency representation in the same species (Suga and Jen, 1976; Suga and Manabe, 1982), we show that these two important parameters, time and frequency, are represented separately in the auditory cortex.

In echolocation, it has been shown that the time interval between an emitted biosonar signal and the returning echo conveys information about the distance to a target (Simmons, 1971, 1973). Variations in the timing of these acoustic events are very important for successful echolocation. Consequently, echo ranging is a suitable model for investigating the processing of time-varying signals in the mammalian auditory system. We have reported previously that the auditory cortex of the mustached bat contains an area (the FM-FM area) where neurons are specialized to respond to combinations of frequency-modulated (FM) signals and presumably are concerned directly with encoding target range information (O'Neill and Suga, 1979; Suga et al., 1978; Suga and O'Neill, 1979). In this paper, we report in much greater detail on the response properties and functional organization of neurons in the FM-FM area in the mustached bat, an area which is not tonotopically organized but contains neurons that encode pulse-echo time intervals related to target range. Furthermore, we provide additional evidence that these neurons are organized along a systematic neural axis representing target range (Suga and O'Neill, 1979).

Most species of bats used in studies of neural range coding are so-called "FM bats," which employ brief, downward-sweeping FM sounds during target approach. The mustached bat, on the other hand, emits trains of more complex biosonar signals, each pulse containing a long, constant frequency (CF) component followed by a brief, downward-sweeping FM component. Each such CF-FM pulse contains up to four harmonics $\left(\mathrm{H}_{1}\right.$ to $\left.\mathrm{H}_{4}\right)$. Of these, the second harmonic at around $61 \mathrm{kHz}$ is predominant (see Fig. 1). The mustached bat holds the frequency of the CF under tight control at a value typical for the individual bat, but, during a process called "Doppler shift compensation," the pulse frequency is reduced in direct proportion to the magnitude of the Doppler shift imparted to the echo by relative motion between the bat and an approaching target (Schnitzler, 1970). In long CF-FM bats, overlap of the CF components of pulse and echo always occurs in target pursuit and in Doppler shift compensation (Schuller, 1974; Schuller and Suga, 1976). CF sounds are excellent for echo detection and especially for analyzing movement, and modulations of the frequency and amplitude of CF portions of the echoes are probably used to sense relative velocity and to detect animate (i.e., moving) targets (Schuller and Suga, 1976; Suga and Jen, 1977; Goldman and Henson, 1977; Pollak, 1980). Bats adjust the repetition rate and duration of their pulses to avoid overlap of the pulse FM components with the echo FM components, probably both to prevent masking and to obtain target information more rapidly when nearing interception. FM sounds are especially suited for localization, ranging, and characterization of a target. In fact, the terminal FM component, as shown for all species so far examined, is used for ranging (Simmons, 1971, 1973). In this paper, we show that processing of different aspects of information derived from the CF and FM components is done in separate regions of the mustached bat cortex.

\section{Materials and Methods}

Sixteen Panamanian mustached bats, Pteronotus parnellii rubiginosus, ${ }^{3}$ were prepared for extracellular recording from single neurons or clusters of two to three neurons in the auditory cortex. In a few initial experiments, surgery was performed under sodium pentobarbital anesthesia (Nembutal, $30 \mathrm{mg} / \mathrm{kg}$, i.p.); in subsequent experiments, the short-acting neuroleptanalgesic Innovar-vet was used $(0.08 \mathrm{mg} / \mathrm{kg}$ of Fentanyl, $4 \mathrm{mg} / \mathrm{kg}$ of droperidol, i.m.), which proved to be much better for the animal's recovery. The large temporal muscles were reflected surgically from their origins on the skull, the skull surface was cleaned, and a $1.5-\mathrm{cm}$ long nail was mounted on the skull using cyanoacrylate glue (Eastman 910) and acrylamide cement. The animal was allowed to recover for at least 2 days prior to the first experiments.

On the day of the experiment, the animal was placed in a double-walled sound-proofed, echo-attenuating chamber after we measured and recorded the frequency of the predominant second harmonic of the bat's biosonar signal. The bat's body was held in a restraint and suspended by elastic bands, while the nail on the skull was clamped in a holder to immobilize the head. The position of the head was adjusted to face the free field speaker. We performed all experiments without general anesthesia, administering the neuroleptic droperidol $(4 \mathrm{mg} / \mathrm{kg})$, when necessary, to reduce excessive movement. Manipulation of surgical wounds was preceded by application of local anesthetic (lidocaine). Throughout the experiment, the bats were given water liberally and sometimes fed mashed mealworms. Nearly all bats were used for a series of 6- to 12-hr recording sessions over a period of 1 to 6 weeks.

All recordings of neural activity were carried out using sharpened tungsten wire microelectrodes $(5-$ to $10-\mu \mathrm{m}$ tips) inserted by hydraulic microdrive into the cortex through small holes bored into the skull. An indifferent electrode was placed in contact with the dura mater through another hole distal to the recording site. Neuronal discharges were amplified (Grass P15), filtered (0.6 to $6.0 \mathrm{kHz}$ bandpass, $24 \mathrm{~dB}$ /octave), and displayed on the screen of an oscilloscope. Action potentials exceeding an adjustable threshold voltage triggered a pulse generator (Tektronix 161), and the resulting uniform pulses were applied to the digital input of a signal processor (Nicolet

\footnotetext{
${ }^{3}$ Pteronotus parnellii rubiginosus $=$ Chilonycteris rubiginosa (Smith, 1972).
} 


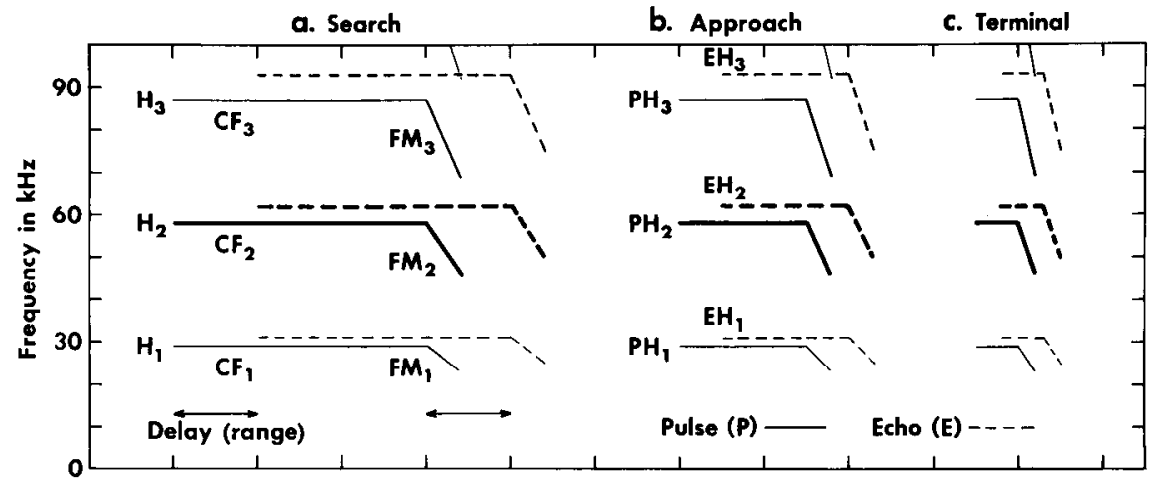

10 msec per division

Figure 1. Schematized sonagrams of the synthesized mustached bat biosonar pulses ( $P$; solid lines) and echoes ( $E$; dashed lines) mimicking three phases of target-oriented flight. The three harmonics of both the pulses $\left(P H_{1-3}\right)$ and the echoes $\left(E H_{1-3}\right)$ each contain a long CF component $\left(C F_{1-3}\right)$ followed by a short FM component $\left(F M_{1-3}\right)$. The fourth harmonic $\left(H_{4}\right)$ is not shown in the figure. $a$, Search phase; CF and FM durations are 30 and $4 \mathrm{msec}$, respectively. $b$, Approach phase; CF and FM durations are 15 and 3 msec. $c$, Terminal phase; CF and FM durations are 5 and 2 msec. Repetition rates for $a, b$, and $c$ were always 10,40 , and $100 \mathrm{pairs} / \mathrm{sec}$, respectively. The thickness of the lines indicates the relative amplitudes of each harmonic in the pulses and echoes: $H_{2}$ is strongest, followed by $H_{3}(-6 \mathrm{~dB})$ and $H_{1}(-12 \mathrm{~dB})$. Echo delay is measured as the time interval between the onsets of corresponding components of the pulse and the echo in a stimulus pair. (From O'Neill and Suga, 1979; used with permission from American Association for the Advancement of Science.)

1072). These same pulses were amplified by an audio monitor and also were used to modulate the brightness of the oscilloscope trace displaying recorded impulses, allowing audiovisual verification of the impulses being processed by the computer. Responses often were quantified from peristimulus time (PST) histograms generated by the computer.

Stimulation. The stimulus delivery system consisted of a voltage-controlled waveform generator (Wavetek 115 or 144), variable duration and rise/fall time electronic switch, and decade attenuator (Hewlett-Packard 350D). These produced shaped tone bursts (rise/fall times, 0.5 msec) in the usual fashion. Two such systems were employed to generate pairs of tone bursts simulating pulses and echoes. These were mixed, amplified (KrohnHite), and broadcast from a 2-inch-diameter electrostatic loudspeaker $( \pm 3 \mathrm{~dB}$ from 20 to $130 \mathrm{kHz}$ ) located $66 \mathrm{~cm}$ in front of the animal's head. All stimulus amplitudes were measured by a calibrated $1 / 4$-inch microphone (Brüel and Kjaer 4135) placed at the same location as the bat's head would be in the recording apparatus, and sound levels are expressed in decibels, sound pressure level $(\mathrm{dB}$ SPL; sound pressure level re: $2 \times 10^{-5} \mathrm{~Pa}$ ).

Inserted between the oscillator and electronic switch in each of these systems was a circuit which multiplies the oscillator frequency to generate the second and third harmonics. Therefore, we could produce stimulus pairs containing one, two, or three harmonics, in any combination, to mimic the harmonic structure of the bat's biosonar signal. Complex or simple stimuli of this sort could be used to analyze each neuron's response selectivity.

To simulate the FM component of the bat's signal, we applied a ramp voltage to the voltage-controlled generator input of the wave generator to produce a linear FM sweep. We restricted the bandwidth of the FM sweep to values similar to natural biosonar signals: $6,12,18$, and $24 \mathrm{kHz}$ for the four possible harmonics.

Whenever we delivered stimulus pairs that included all three harmonics, the amplitude ratios among the harmonics of the individual stimuli were set at values typical of mustached bat pulses; i.e., the first and third harmonics were, respectively, 12 and $6 \mathrm{~dB}$ lower than the predominant second harmonic.

To simulate more accurately the dynamic conditions of target pursuit, we produced these synthesized pulseecho stimuli at three repetition rates and durations typical of the search, approach, and terminal phases of mustached bat echolocation (Table I).

Approach and terminal phase stimuli were gated to produce trains of about $500 \mathrm{msec}$, repeated one per sec, to reduce neural "fatigue." In nature, the durations of the approach and terminal phases are typically 180 and 50 msec, respectively (Novick and Vaisnys, 1964).

Because of the complexity of mustached bat biosonar signals, as well as the stimuli used in the study to mimic their signals, we have introduced a shorthand notation to refer to the various components in these sounds (Suga et al., 1978). These are depicted in Figure 1, which shows pairs of sounds referred to as "pulses" $(P)$ or "echoes" $(E)$. A pulse produced by a bat may consist of up to four harmonics, $\mathrm{H}_{1-4}$, each of which always contains a constant frequency (CF) component followed immediately by a frequency-modulated (FM) component. The individual $\mathrm{CF}$ and FM components of each harmonic are appropriately subscripted (e.g., $\mathrm{FM}_{2}$ is the FM component of $\mathrm{H}_{2}, \mathrm{CF}_{1}$ is the $\mathrm{CF}$ component of $\mathrm{H}_{1}$ (the fundamental), and so on). In a similar fashion, an echo also may contain up to four harmonics, which also can be referred to as $\mathrm{CF}_{1-4}-\mathrm{FM}_{1-4}$ (i.e., $\mathrm{H}_{1-4}$ ) or simply as "E." In all cases, where we needed to distinguish between pulse and echo components, we used prefixes "P" or "E." In our papers, "P-E" always indicates a pair of CF-FM sounds that are harmonically complex like naturally occurring pulses and echoes. $\mathrm{P}$ and $\mathrm{E}$ are usually also distinguished by their differences in frequency due to Doppler shifts in the echo and by their relationship in time. 
'IABLE' I

Stimulus repetition rates and durations mimicking the search, approach, and terminal phases of mustached bat echolocation.

\begin{tabular}{lrrr}
\hline & \multirow{2}{*}{ Repetition Rate } & \multicolumn{3}{c}{ Duration } \\
\cline { 3 - 4 } & & CF & FM \\
\hline & & & msec \\
Search & & 30 & 4 \\
Approach & $40 / \mathrm{sec}$ & 15 & 3 \\
Terminal & $100 / \mathrm{sec}$ & 5 & 2 \\
\hline
\end{tabular}

We refer to our stimuli using the same notation except that we are able to add or subtract entire harmonics or individual CF or FM components using the controls of the harmonic generator previously described. We are restricted to being able to generate a maximum of only three harmonics at once, however. A complete P-E pair of stimuli consisted of only $\mathrm{PH}_{1-3}-\mathrm{EH}_{1-3}$ in our experiments. Less complex stimuli are more specifically designated. For example, $\mathrm{PH}_{1,3}-\mathrm{EH}_{2}$ would refer to a stimulus pair containing the $\mathrm{H}_{1}$ and $\mathrm{H}_{3}$ components in the initial pulse stimulus and only the $\mathrm{H}_{2}$ components in the echo stimulus. Thus, whenever a "P" or " $E$ " is used without further elaboration, it always implies that a complete, three-harmonic CF-FM stimulus was used, but when the $\mathrm{P}$ or $\mathrm{E}$ is followed by specific references to components, we state explicitly those components used in this more simplified stimulus. The simplest stimuli used are sounds containing a single $\mathrm{CF}\left(\mathrm{CF}_{1}, \mathrm{CF}_{2}\right.$, etc. $)$ or $\mathrm{FM}$ component $\left(\mathrm{FM}_{1}, \mathrm{FM}_{2}\right.$, etc. $)$.

Measurement of neural responses to such stimuli was performed as follows. Our search stimulus consisted of P-E pairs with echo delays anywhere from 1 to $20 \mathrm{msec}$. When a neuron was encountered, we gradually simplified this stimulus by eliminating the individual harmonics in both $\mathrm{P}$ and $\mathrm{E}$. This process of elimination eventually demonstrated which components were essential to elicit the neural response at the lowest threshold. These sounds are called the essential components for response. The neurons were thereby classified according to their essential components; e.g. $\mathrm{FM}_{1}-\mathrm{FM}_{3}$ neurons responded best to a pair of stimuli containing at the very least $F M_{1}$ and $\mathrm{FM}_{3}$. Addition of other harmonics to these essential components resulted in either no change in response or only a slight modification of response magnitude, as will be indicated in the results. This demonstrates the flexibility of this system; complex, biologically realistic stimuli can be generated and then simplified to determine the selectivity of a neuron to the individual sound elements.

To study the neural responses at different pulse-echo time intervals, we first set both the pulse and echo stimulus at their respective best frequencies for facilitation. Then, while holding the pulse amplitude constant, usually at its best amplitude for facilitation, we varied the echo amplitude and its delay from the pulse to measure the response. In many cases, there was no best amplitude for facilitation for the pulse, because the facilitation that it produced when paired with the echo reached a plateau of maximum response just above threshold (Suga et al., 1978). Therefore, no single best amplitude could be defined, and the pulse amplitude used was somewhat arbitrary since any value on the plateau had the same magnitude of effect. We usually set pulse amplitude at a high value in these cases (60 to 80 dB SPL), to mimic the strong stimulation of the bat's ears by its own emitted biosonar signal.

Mapping. As will be shown, a large majority of the neurons that we recorded were delay tuned; i.e., they responded to echo stimuli following pulse stimuli only at certain time delays. The pulse-echo delay generating the lowest threshold for the echo stimulus is called the neuron's best delay (BD). We proceeded to map the BDs of these neurons in the cortex, in the same way that one measures the distribution of neuronal best (characteristic) frequencies.

In the initial mapping experiments, microelectrodes were inserted perpendicular to the cortical surface, and the $\mathrm{BD}$ was measured for recording sites at depths between 150 and $1000 \mu \mathrm{m}$ (responses above and below these depths are uniformly poor or hard to isolate). In some experiments, not only the $\mathrm{BD}$ but also the change in threshold as a function of echo delay, i.e., the delay. tuning curve, was measured for each neuron.

Once we had demonstrated that BD was uniform for a particular vertical penetration (i.e., that neurons were organized into columns according to best delay), we then proceeded to map variation in $\mathrm{BD}$ among columns by passing the electrode oblique to the cortical surface. Electrodes were directed either dorsoventrally or rostrocaudally. Responses usually were sampled at $200-\mu \mathrm{m}$ intervals. Results of each penetration were plotted on a map of the cortical surface. For standardization, all maps were referred to the left cerebral hemisphere even though mapping was carried out bilaterally.

\section{Results}

Spectral selectivity of combination-sensitive neurons in the FM-FM area. Although we have chosen to describe elsewhere the details of the unique frequencyselective features of neurons in the FM-FM area (Suga et al., 1978, 1981; N. Suga and W. E. O'Neill, manuscript in preparation), it is essential at the outset to summarize these properties in order to provide a better understanding of the range sensitivity of these neurons to be discussed subsequently.

The neurons described in this paper were recorded from a $3-\mathrm{mm}^{2}$ area dorsorostral to the tonotopically organized auditory field described by Suga and Jen (1976). In stark contrast to the neurons of the tonotopically organized area, the majority of neurons here responded poorly, or did not respond, to pure tone bursts at amplitudes less than $80 \mathrm{~dB}$ SPL. Noise bursts (NB) and FM sounds whose durations and bandwidths were adjusted to be roughly equivalent to the FM components of the mustached bat biosonar signal were equally ineffective when presented alone. These neurons responded vigorously, however, to pairs of stimuli which mimic pulses and echoes by containing the first three harmonics of the biosonar signal (Suga et al., 1978, 1981).

By systematically eliminating the various CF and FM components from each stimulus in a pair, we found that the most effective combinations, or essential components for "facilitation," were the $\mathrm{FM}_{1}$ of the initial stimulus (simulated pulse, $\mathrm{P}$ ) and either the $\mathrm{FM}_{2}, \mathrm{FM}_{3}$, or $\mathrm{FM}_{4}$ of 
the second stimulus (simulated echo, E). There was never facilitation when the $\mathrm{FM}_{1}$ component was deleted from the pulse; neither was there a response to pairs consisting only of $\mathrm{PFM}_{1}-\mathrm{EFM}_{1}$. This selectivity was unaffected by the addition of CF components at any frequency, intensity, and duration. The responses to stimulus pairs containing the essential components consisted of a single burst of impulses to each pair, following the echo FM component (EFM) with a latency of 5 to $10 \mathrm{msec}$, depending on the neuron. Since the response to the echo component was facilitated by the presence of a preceding $F M_{1}$, we have called these neurons collectively $F M_{1}-F M_{n}$ facilitation neurons (where $n=2,3$, or 4; Suga et al., 1978; O'Neill and Suga, 1979; Suga and O'Neill, 1979).

$\mathrm{FM}_{1}-\mathrm{FM}_{2}$ neurons are selective for a pair of sounds: the first sweeping downward from about 30 to $24 \mathrm{kHz}$ $\left(\mathrm{FM}_{1}\right)$ and the second sweeping from about 62 to $50 \mathrm{kHz}$ $\left(\mathrm{FM}_{2}\right.$; the exact frequency values of the best $\mathrm{FM}$ stimuli are different for each neuron although the bandwidths of the stimuli were held constant). Likewise, $\mathrm{FM}_{1}-\mathrm{FM}_{3}$ neurons are selective for an echo FM sweeping typically from about 93 to $75 \mathrm{kHz}$, and $\mathrm{FM}_{1}-\mathrm{FM}_{4}$ neurons are selective for an echo FM sweeping from about 124 to 100 $\mathrm{kHz}$. The frequency relationships of the $\mathrm{PFM}_{1}$ and $\mathrm{EFM}_{n}$ were usually nonharmonic, and in nearly all cases, the best facilitation frequency of the $\mathrm{FM}_{1}$ stimulus was lower than the frequency predicted by calculating the fundamental of the essential echo $\mathrm{FM}_{n}$ component. This may well be an adaptation to Doppler shift compensation which is used by this species to keep the echo $\mathrm{CF}_{2}$ component from moving targets in a narrow frequency band around $61 \mathrm{kHz}$ (Schnitzler, 1970; Schnitzler and Henson, 1980; W. E. O'Neill and N. Suga, unpublished observations).

As we have already implied, a particular time delay between the $\mathrm{PFM}_{1}$ and $\mathrm{EFM}_{n}$ stimuli is necessary to elicit maximal facilitation of response. This sensitivity to time interval variations led us to investigate systematically the responses of $\mathbf{F M}_{1}-\mathrm{FM}_{n}$ neurons to changes in pulse-echo delay.

Responses of $F M_{1}-F M_{n}$ facilitation neurons as a function of echo delay. By delivering either or both of the essential components, or all three harmonics of simulated pulse and echo stimuli, and by varying the time interval between them, we measured the responses of $405 \mathrm{FM}_{1}$ $\mathrm{FM}_{n}$ neurons to conditions mimicking targets at different distances.

To illustrate the way in which neurons in the FM-FM area responded to different time delays between pulse and echo, PST histograms of the response of an $\mathrm{FM}_{1-}$ $\mathrm{FM}_{2}$ facilitation neuron are shown in Figure 2. As illustrated by the histograms in the top two ranks of the figure, the neuron did not respond when presented with $\mathrm{P}$ or $\mathrm{E}$ alone at any of the three repetition rates $(A=10 /$ sec; $B=40 / \mathrm{sec} ; C=100 / \mathrm{sec})$. When $\mathrm{P}$ and $\mathrm{E}$ were presented as a pair of stimuli with no time interval between them at a rate of $10 / \mathrm{sec}(\mathrm{E}$ delay $=0 \mathrm{msec})$, there was also no response (Fig. $2 A$ ). This holds also for the other two repetition rates. However, when $\mathrm{E}$ was progressively delayed from $P$, responses to the pairs became stronger as a function of echo delay, reaching a maximum at 6.5 to $7.0 \mathrm{msec}$ echo delay. At $10 / \mathrm{sec}$,

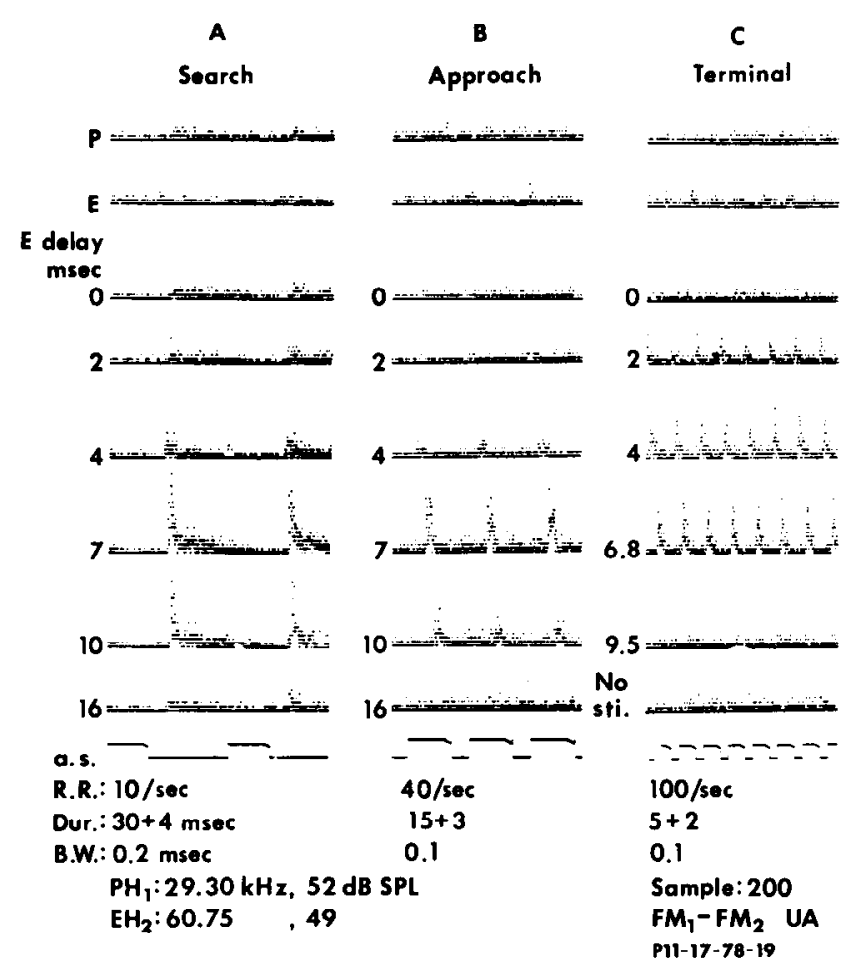

Figure 2. Peristimulus time (PST) histograms of the response of an $\mathrm{FM}_{1}-\mathrm{FM}_{2}$ facilitation neuron to pulse-echo stimulus pairs as a function of echo delay. Stimuli were delivered at repetition rates and durations simulating the search $(A, 10 / \mathrm{sec})$, approach $(B, 40 / \mathrm{sec})$, and terminal phases $(C, 100 / \mathrm{sec})$. PSTs in the first two ranks in each condition show the responses to the complete (three harmonics) pulse $(P)$ and the complete echo $(E)$ presented alone. All others show the responses to pairs of $\mathrm{P}$ and $\mathrm{E}$ sounds at various echo delays. Maximum responses occur at 6.8 to $7.0 \mathrm{msec}$ echo delay at each repetition rate. a.s., time markers of the pulse in the stimulus or stimulus pairs; $R . R$., repetition rate of stimulation; Dur., duration of the CF and FM components, respectively; $B . W$., bin width in milliseconds of the PST histograms; Sample, the number of stimulus repetitions presented for each PST histogram. $U A$ indicates an unanesthetized preparation. Frequency and amplitude of essential harmonics are given at the bottom. No sti., histogram of spontaneous activity (no stimulus).

responses could be detected at echo delays from 2 to 16 msec at this stimulus amplitude (49 dB SPL re: $\mathrm{EH}_{2}$ ).

In response to shorter duration stimulus pairs delivered at $40 / \mathrm{sec}$ (approach phase, Fig. $2 B$ ), the maximum response elicited from this neuron was again at an echo delay of $7.0 \mathrm{msec}$. When compared to the responses at $10 / \mathrm{sec}$, there is some loss of responsiveness, or a narrowing of the temporal selectivity, at delays of 2 or $16 \mathrm{msec}$.

At 100 stimulus pairs/sec (terminal phase, Fig. 2C), the neuron still responded with extraordinary vigor, with a response maximum at $6.8 \mathrm{msec}$ echo delay. Some shifting of the range of the echo delays eliciting responses to shorter values is evident from the increase in responses to $4 \mathrm{msec}$ echo delay and the decrease in response at 9.5 msec echo delay when compared to 10 or $40 /$ sec. Nevertheless, the echo delay eliciting greatest response remained at 6.8 to $7.0 \mathrm{msec}$ at all three repetition rates and corresponding stimulus durations.

The P-E stimulus pairs contained a total of six CF and 
FM components. However, the essential components for facilitation of this neuron were found to be $\mathrm{PFM}_{1}$ and $\mathrm{EFM}_{2}$. The best $\mathbf{F M}_{1}$ and $\mathrm{FM}_{2}$ for facilitation swept from 29.30 to $23.30 \mathrm{kHz}$ and from 60.75 to $48.75 \mathrm{kHz}$, respectively. At these frequencies, the best $\mathrm{EH}_{2}$ was $2.15 \mathrm{kHz}$ greater than $\mathrm{PH}_{2}(29.3 \times 2=58.6 \mathrm{kHz})$; i.e. the neuron responded best to an echo Doppler-shifted by an approaching target. The measured resting frequency (no Doppler compensation) of this bat was 60.3 to $60.8 \mathrm{kHz}$. This neuron would be most active for echoes for which compensation had already occurred.

Also important for this response was the amplitude relationship between $\mathrm{PFM}_{1}$ and $\mathrm{EFM}_{2}$. For maximum response, the amplitudes of $\mathrm{PFM}_{1}$ and $\mathrm{EFM}_{2}$ were 52 and $49 \mathrm{~dB}$ SPL, respectively. No facilitation occurred when these components were made much more intense.

Figure 3 illustrates the responses of a different $\mathbf{F M}_{1}$ $\mathrm{FM}_{2}$ neuron in which maximum facilitation occurred at a much shorter echo delay. In this experiment, only the essential harmonics, $\mathrm{PH}_{1}$ and $\mathrm{EH}_{2}$, were presented. As in the aforementioned neuron of Figure 2, this neuron did not respond when $\mathrm{PH}_{1}$ or $\mathrm{EH}_{2}$ was presented alone regardless of amplitude, duration, and repetition rate, nor did it respond to $\mathrm{PH}_{1}-\mathrm{EH}_{2}$ pairs with 0 msec delay. When a delay between $\mathrm{PH}_{1}$ and $\mathrm{EH}_{2}$ was introduced, however, the neuron responded to each pair. The maximum response was obtained at 4 to $5 \mathrm{msec}^{\mathrm{EH}} \mathrm{H}_{2}$ delay. As repetition rate increased, there was some shrinking of

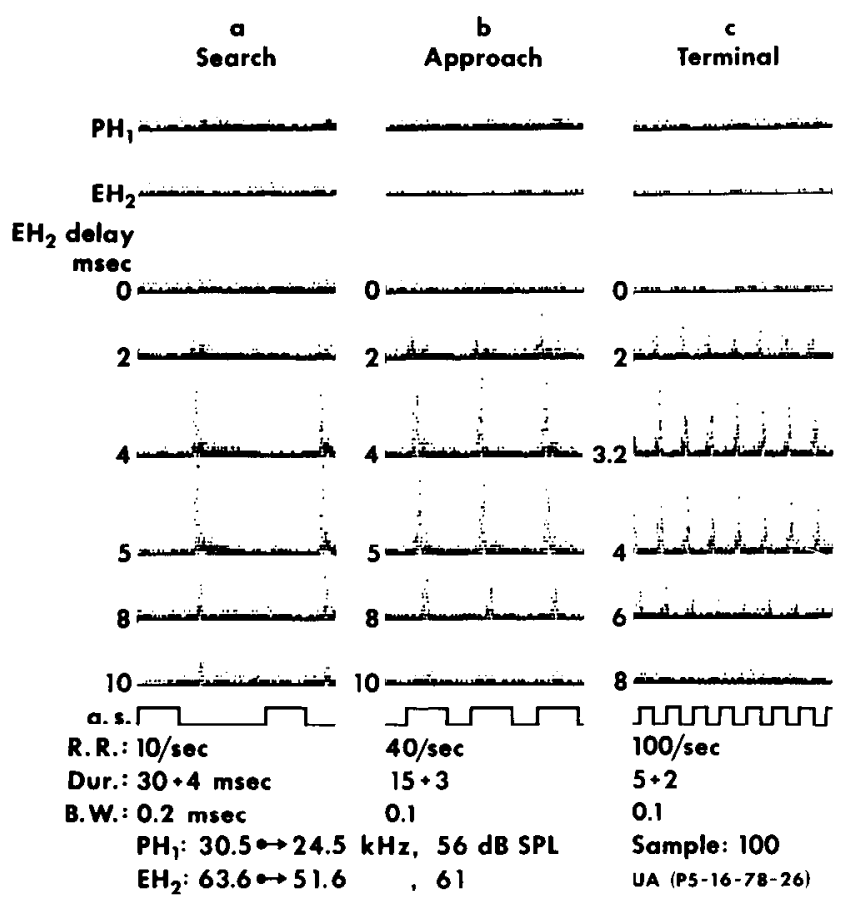

Figure 3. PST histograms of the responses of an $\mathrm{FM}_{1}-\mathrm{FM}_{2}$ neuron selective for echo delays of 3.2 to $4.0 \mathrm{msec}$. Note the similarity of the response of this neuron to that shown in Figure 2 , except that the echo delay eliciting the maximum response is shorter. In this case, the number of stimulus presentations was 100 for each histogram, and only the essential harmonics, $\mathrm{PH}_{1}$ and $\mathrm{EH}_{2}$, are used as stimuli. Stimulus frequency and amplitude of the essential harmonics are given at the bottom of the figure. The abbreviations used are defined in the legend to Figure 2. the range of delays to which the unit responded, and maximum response shifted slightly to $3.2 \mathrm{msec}$.

As was the case in the neuron described in Figure 2, this neuron also is "preadapted" to respond to Dopplershifted echoes. In the neuron of Figure 3, the best $\mathrm{EFM}_{2}$ was $2.6 \mathrm{kHz}$ greater than the calculated $\mathrm{PH}_{2}$. Measured resting frequency of the bat was 60.7 to $61.0 \mathrm{kHz}$. In contrast to the previous neuron, which would fire maximally when the bat had already compensated for the echo Doppler shift, this neuron would be most likely to respond to echoes prior to compensation when the bat was still emitting pulses near its resting frequency.

As shown in Figures 2 and $3, \mathrm{FM}_{1}-\mathrm{FM}_{n}$ neurons commonly produced discharges which were well synchronized to the time course of the paired stimuli delivered at 100/ sec. However, such stimulus-locked responses are not necessary to decode echo delay. In Figure $6 B$, for instance, the neuron responded best to P-E pairs with a 1.5-msec echo delay in spite of the lack of a clear stimulus-locked discharge pattern. In striking contrast, its response to P-E pairs with 0 - or $4.5-\mathrm{msec}$ echo delays is quite poor.

In order to quantify the change in response of $\mathrm{FM}_{1-}$ $\mathrm{FM}_{n}$ neurons to echo delay, we measured the threshold for facilitation as a function of delay, i.e., a delay-tuning curve, in 43 neurons. Best delay (BD) then could be defined as that echo delay to which the neuron was most sensitive (had the lowest threshold). In this way, we found two different types of delay-sensitive neurons, tracking and delay-tuned neurons (O'Neill and Suga, 1979; Suga et al., 1978). In tracking neurons, both the shape of the delay-tuning curve and the best delay changed as the repetition rate and stimulus durations were changed. Two examples of this are shown in Figure 4. Notice that, in both cases, the delay-tuning curves for both 10 and $40 / \mathrm{sec}$ repetition rates are fairly broad and U shaped. At 100/sec, the curves become much narrower and V shaped. In both of these neurons as well as in nearly all others of this type, the sharpening of the delaytuning curves occurs through a change in sensitivity to echo delays greater than $\mathrm{BD}$, while the curves remain roughly the same for echo delays less than $\mathrm{BD}$. Also typical was an increase in minimum threshold by about $10 \mathrm{~dB}$ at the high repetition rate. In all neurons of this type, where the $\mathrm{BD}$ varied with repetition rate, $\mathrm{BD}$ always became shorter as the repetition rate increased. This characteristic would act to maintain responsiveness in these units to the shortening of echo delay occurring as the bat closes on a target.

The more common delay-tuned neurons had much less variable BDs and sharper delay-tuning curves. Figure 5 shows three examples of delay-tuned neurons. The neuron in Figure $5 A$ had BDs of 1.2 to $1.6 \mathrm{msec}$ at all three standard repetition rates; that is, it is tuned to 1.2to 1.6 -msec echo delays regardless of the repetition rate and duration of the stimuli. The delay-tuning curves are somewhat different for echo delays greater than BD, since they are broader at $10 / \mathrm{sec}$ than at either 40 or 100 / sec. At $100 / \mathrm{sec}$, the tuning is very sharp, and the neuron was clearly nonmonotonic. In fact, upper thresholds appeared in the response; i.e., when the echo was above 72 $\mathrm{dB}$ SPL, the neuron no longer responded. This is called a "closed" tuning curve. In spite of this variability in the 


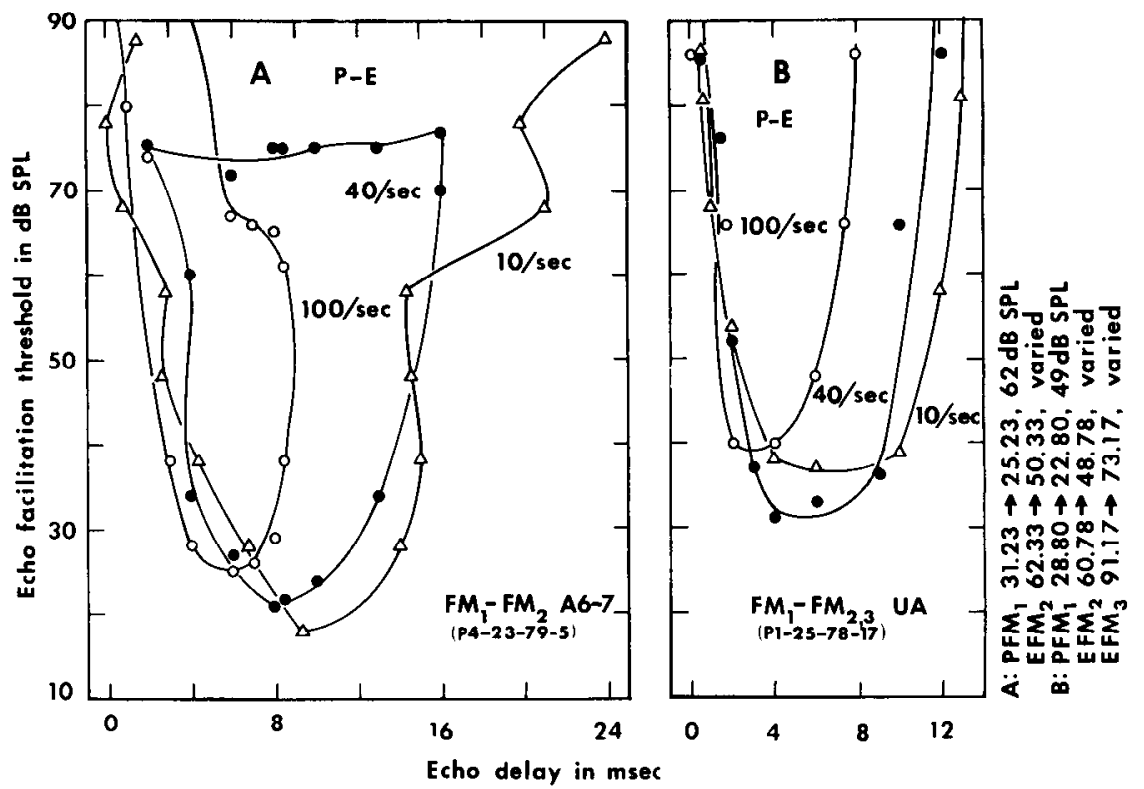

Figure 4. Delay-tuning curves of two tracking neurons. In both cases, measurements of the echo threshold as a function of delay from the pulse stimulus were made using complete P-E stimuli. The three curves in each graph represent the delay-tuning curve measured at repetition rates of $10 / \mathrm{sec}(\triangle), 40 / \mathrm{sec}(O)$, and $100 / \mathrm{sec}(O)$. Frequency and amplitude parameters are given for each on the right of the figure. A6-7 indicates the measurements were made 6 to $7 \mathrm{hr}$ after administration of Innovar-vet. $U A$, unanesthetized preparation.

shape of the curves, we still consider this neuron delay tuned because of the constancy of the $\mathrm{BD}$.

Figure $5 B$ shows a delay-tuned neuron with a constant $\mathrm{BD}$ and nearly similar delay-tuning curves. The $\mathrm{BD}$ of this neuron was 1.5 and $1.2 \mathrm{msec}$ at 40 and $100 / \mathrm{sec}$, respectively. (Measurements were unable to be taken at $10 / \mathrm{sec}$.) BDs this short were nearly always correlated with sharp delay-tuning curves and, very often, closed tuning curves.

Figure $5 C$ illustrates the delay-tuning curves of yet another delay-tuned neuron, whose BDs at 10 and $40 /$ sec were 8 and $7.2 \mathrm{msec}$, respectively. The delay-tuning curves are closed and very similar in shape and are considerably broader than those measured for the neuron in Figure $5 B$. As will be demonstrated elsewhere, this again shows that neurons with longer $\mathrm{BDs}$ also had broader delay-tuning curves. At 100/sec, the response of this neuron (Fig. $5 C$ ) was still evident, despite its long $\mathrm{BD}$, but was so reduced that a delay-tuning curve could not be measured with accuracy. The area in which clear responses were seen is shown by the hatched rectangle in the figure, located at a delay of $7.5 \mathrm{msec}$ and amplitudes between 40 and $50 \mathrm{~dB}$ SPL. Very often, neurons with long BDs showed this type of response at $100 / \mathrm{sec}$; others were found with BDs longer than 9 to $10 \mathrm{msec}$, which did not respond at all to stimuli at this rate.

Even though there are distinct differences among the delay-tuning curves of different neurons, the common characteristic of delay-tuned neurons is the stability of both the BDs and the shapes of the curves at different repetition rates. Our operational definition for calling a neuron "delay tuned" was that its $\mathrm{BD}$ varied $1 \mathrm{msec}$ or less at the three repetition rates used in our study. However, delay-tuned neurons with long BDs responded poorly, if at all, to stimulus pairs at 100/sec (e.g., Fig. $5 C$ ). In most cases, this was because, at this rate, the time interval between repetitions of the $P$ stimulus $(10 \mathrm{msec})$ was shorter than the BD. At $100 / \mathrm{sec}$, for example, an echo delayed by $12 \mathrm{msec}$ from the pulse is also actually 2 msec delayed from the subsequent pulse. If the neuron's $\mathrm{BD}$ is $12 \mathrm{msec}$, then a $2-\mathrm{msec}$ echo delay elicits no response. This problem can be avoided only by lowering the repetition rate or shortening the pulses. As we have mentioned, bats carefully avoid overlap of pulse and echo FM components. Since small targets must be as close as $50 \mathrm{~cm}$ (echo delay, $2.9 \mathrm{msec}$ ) before the mustached bat begins the terminal phase buzz, responding at high repetition rates is normally irrelevant to neurons with long BDs. In these cases, if the BD varied by 1 msec or less for 10 and $40 / \mathrm{sec}$ stimulation, the neuron also was classified as delay tuned.

The inclusion of the nonessential components in complete $\mathrm{P}-\mathrm{E}$ stimulus pairs did not alter the $\mathrm{BD}$ appreciably, but it sometimes reduced the magnitude of facilitation observed when only the essential components were employed. We therefore wondered what differences the nonessential components might produce in the overall delay sensitivity of these neurons. The PST histograms of Figure 6 illustrate the responses of a neuron to different echo delays when presented with P-E pairs (Fig. 6, $A$ and $B$ ) as compared to pairs of the essential components, $\mathrm{PH}_{1}-\mathrm{EH}_{2}$, only (Fig. $6 \mathrm{C}$ ). At $10 / \mathrm{sec}$ repetition rate, the response is already quite diminished at only $3.5 \mathrm{msec}$ echo delay for the P-E pairs $(A)$ in contrast to the clear responses at this delay when using only the essential components $(C)$. The excitation produced by complete $\mathrm{P}$ or $\mathrm{E}$ stimuli alone $(A, t o p)$ is also noticeably less than that produced by $\mathrm{PH}_{1}$ or $\mathrm{EH}_{2}$ stimuli alone $(C, t o p)$. The $\mathrm{BD}$ of this neuron is very short, $1.5 \mathrm{msec}$. Consequently, facilitation is noticeable at the suprathreshold echo amplitude employed (54 dB SPL for $\mathrm{EH}_{2}$ ) even at $0 \mathrm{msec}$ delay.

Figure 7 illustrates the changes that complete P-E pairs can bring about in the delay-tuning curves. In each 


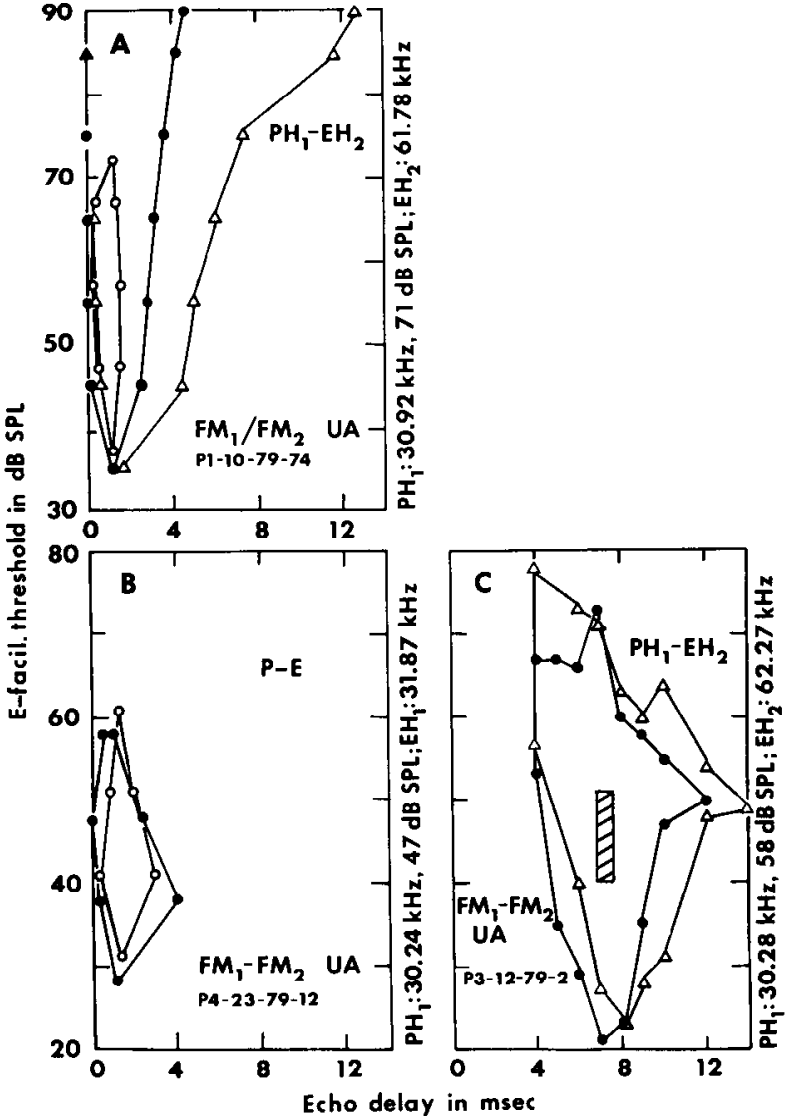

Figure 5. Delay-tuning curves of three delay-tuned neurons. In these neurons, $\mathrm{BD}$ varies by no more than $1 \mathrm{msec}$ at different repetition rates. All are $\mathrm{FM}_{1}-\mathrm{FM}_{2}$ facilitation neurons. Values of frequency and amplitude of stimulus pairs used to measure the curves are to the right of each figure. Data at $10 / \mathrm{sec}$ were not obtained for the neuron in $B$ because its BD was $0.8 \mathrm{msec}$ nor at $100 / \mathrm{sec}$ for the neuron in $C$ because its response at this rate was so poor. The hatched rectangle indicates the echo delay and amplitude eliciting the greatest responses. $\Delta, 10 / \mathrm{sec}$ repetition rate;, $40 / \mathrm{sec}$ repetition rate; $O, 100 / \mathrm{sec}$ repetition rate. $U A$, unanesthetized preparation.

case, the complete P-E pairs generated a delay-tuning curve which was sharper than its counterpart measured only with pairs of essential components, both at $40 / \mathrm{sec}$ (Fig. 7, $A 1$ and $B 1$ ) and at 100/sec (Fig. 7, $A 2$ and B2). Even more noticeable than the change in sharpness is the shrinking of the dynamic range for echo amplitude: the neurons had higher thresholds and were much more strongly nonmonotonic using P-E pairs. Thus, under presumably more natural multiharmonic stimulus configurations, these neurons become more selective not only for target range but also for echo amplitude (related to subtended target angle). The two cases shown here are examples of delay-tuned neurons tuned to nearby (Fig. 7, $A 1$ and $A 2 ; \mathrm{BD}=1.5 \mathrm{msec}$ ) and moderately distant (Fig. 7, $B 1$ and $B 2 ; \mathrm{BD}=4.3 \mathrm{msec}$ ) targets.

Many delay-tuned neurons exhibited nonmonotonic responses and even upper thresholds to echo amplitude (e.g., Figs. $4 A$, 5, and 7). In Figure 8, we demonstrate this phenomenon quantitatively in two neurons by plotting the isoimpulse count contours, which connect values of delay and amplitude of the echo eliciting equivalent numbers of action potentials.

The neuron of Figure $8 B$ was stimulated using complete $\mathrm{P}$ and $\mathrm{E}$ pairs. This neuron's response contours show strong amplitude tuning, with a clear best amplitude (BA) for facilitation at about $40 \mathrm{~dB}$ SPL. In this case, the delay associated with maximum response at $\mathrm{BA}$ is the same as the $\mathrm{BD}, 7.0 \mathrm{msec}$. At $40 \mathrm{~dB} \mathrm{SPL}$, the delay-tuning curve is rather broad, extending nearly to 10 msec. Notice, however, the sharp decline in response magnitude at $40 \mathrm{~dB}$ SPL for echo delays less than $7 \mathrm{msec}$. This sharp cut-off is an excellent example of how even neurons with long BDs can be highly sensitive to small changes in echo delay.

Figure $8 A$ shows a neuron tuned to 3.5 msec delay. The contours circumscribe a peak of maximum response (average 2.0 impulses/stimulus pair) at an echo amplitude of $40 \mathrm{~dB}$ SPL and an echo delay of $2.0 \mathrm{msec}$. Thus, $40 \mathrm{~dB}$ SPL can be considered the neuron's best amplitude for facilitation. It is interesting to note that, in this case, the $\mathrm{BD}$ as defined by the neuron's minimum threshold is different from the delay eliciting maximum response at the unit's BA. This characteristic in the delay tuning is not unique to this neuron alone, since two others out of eight whose responses were measured in this way also exhibited this shift. In these cases, the delay eliciting
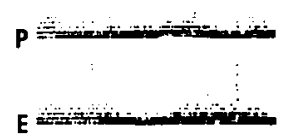

P - E

E delay
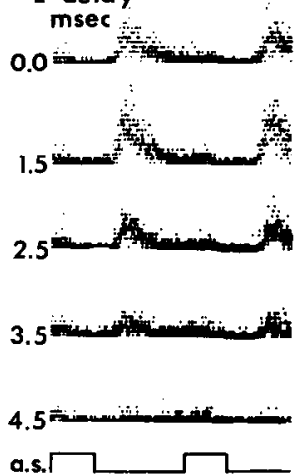

R.R.: $10 / \mathrm{sec}$

$F M_{1}: 30.24 \rightarrow 24,24 \mathrm{kH}$

$F M_{2}: 63.73 \rightarrow 51.73 \quad, 54$

Samp.: 200

Figure 6. PST histograms showing the sharpening of delay tuning in delay-tuned neurons when presented with complete P.E pairs versus pairs containing only essential harmonics. $A$ $(10 / \mathrm{sec})$ and $B(100 / \mathrm{sec})$ sequences were obtained using complete P-E pairs. Maximum response is obtained at $1.5 \mathrm{msec}$ echo delay at both repetition rates. Facilitation responses are no longer visible at delays more than 3.5 msec. In contrast, stimulus pairs $(10 / \mathrm{sec})$ with only essential harmonics $\left(\mathrm{PH}_{1}\right.$ $E H_{2}$ ) elicit stronger responses over a broader echo delay range, well beyond $4.5 \mathrm{msec}$ delay $(C)$. The delay eliciting maximum response remains the same as with $\mathrm{P}-\mathrm{E}$ pairs $(1.5 \mathrm{msec})$. Responses to stimuli presented alone are presented in the top two ranks of the histograms. $N=200$ samples/histogram. The abbreviations used are defined in the legend to Figure 2. 


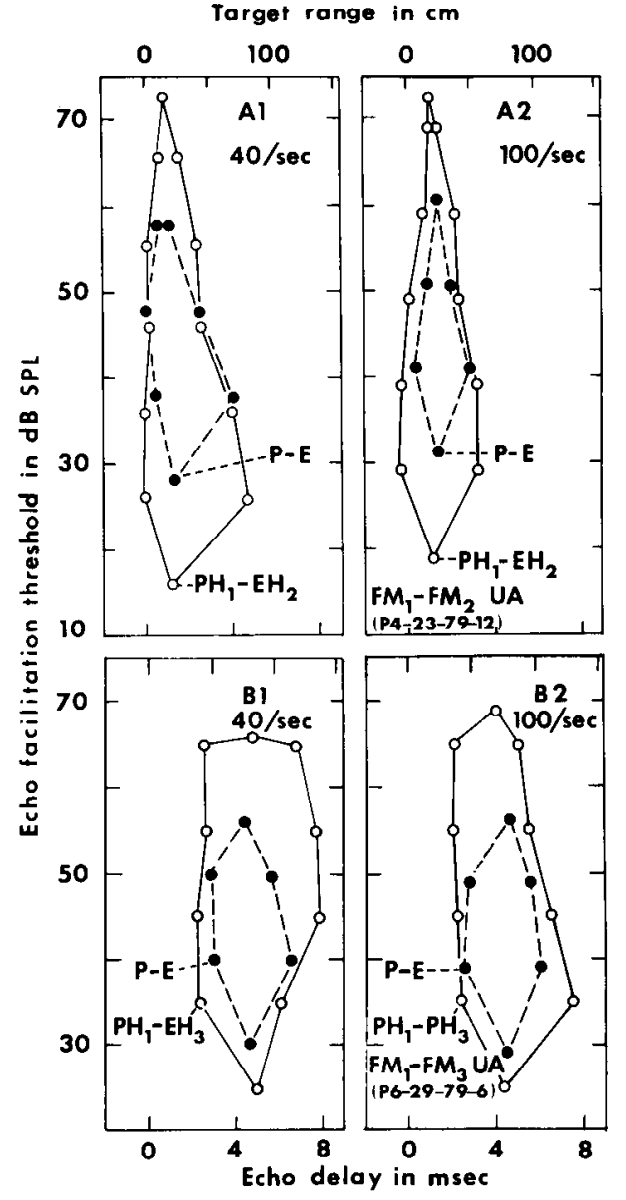

Figure 7. Changes induced in delay-tuning curves of two delay-tuned neurons by presenting complete P-E stimulus pairs versus pairs with only essential harmonics. In both cases ( $A$ and $B$ ), the delay-tuning curves of each neuron were altered, with $\mathrm{P}-\mathrm{E}$ pairs (dashed lines) reducing the range of response in the amplitude domain when compared to curves measured using essential harmonics $\left(P H_{1}-E H_{2}\right.$ for $A ; P H_{1}-E H_{3}$ for $\left.B\right)$. "Bandwidth" of the response to echo delay was altered only minimally; however, these changes always were in the direction of reducing the bandwidth (increasing sharpness) when stimulated with P-E pairs. Repetition rates were $40 / \mathrm{sec}$ in $A 1$ and $B 1 ; 100 / \mathrm{sec}$ in $A 2$ and $B 2 . U A$, unanesthetized preparation.

maximum firing at $\mathrm{BA}$ was shorter than the $\mathrm{BD}$ as defined at threshold. The contours showing moderate responses at higher echo amplitude ( $>60 \mathrm{~dB}$ SPL) at about the same delays are due to the response to the pulse or echo alone.

As previously noted, we observed a general trend for the delay-tuning curves of neurons with long BDs to be not as sharply tuned in time as those with short BDs. Figure 9 is a composite of the delay-tuning curves of seven different range-tuned neurons illustrating how a population of such cells could decode range. Clearly, during target approach, each of these neurons would be activated in succession, beginning with the neuron tuned to $12 \mathrm{msec}$ echo delay (about $206 \mathrm{~cm}$ ). However, a given delay-tuning curve overlaps noticeably with others having different BDs. Echoes with a particular delay therefore activate neurons with different BDs.

The distribution of delay-tuned neurons from all bats as a function of their BDs (Fig. 10) demonstrates clearly a disproportionate number of neurons with BDs between 3 and $8 \mathrm{msec}$ (50 to $140 \mathrm{~cm}$ ). BD varied in the sample from as long as $18 \mathrm{msec}$ to as short as $0.4 \mathrm{msec}$. This correlates well with target ranges appropriate to approach and early terminal phase (Novick and Vaisnys, 1964). However, it must be said that neurons with very short BDs are probably under-represented in our sample because of their relative inaccessibility to our electrode placements. The basis for this statement will be described in the next section.

Distribution of delay-tuned neurons in the FM-FM area: Odotopic representation. Evidence such as we presented in Figures 9 and 10 provided the impetus to explore the anatomical distribution of delay-tuned neurons in the FM-FM area. The first step in this mapping study was to determine how BDs and delay-tuning curves varied with cortical depth. We thus introduced our microelectrodes perpendicular to the cortical surface and recorded BDs and curves at different depths (a companion paper detailing the complex nontonotopic projections of stimulus frequency to the FM-FM area is in preparation).

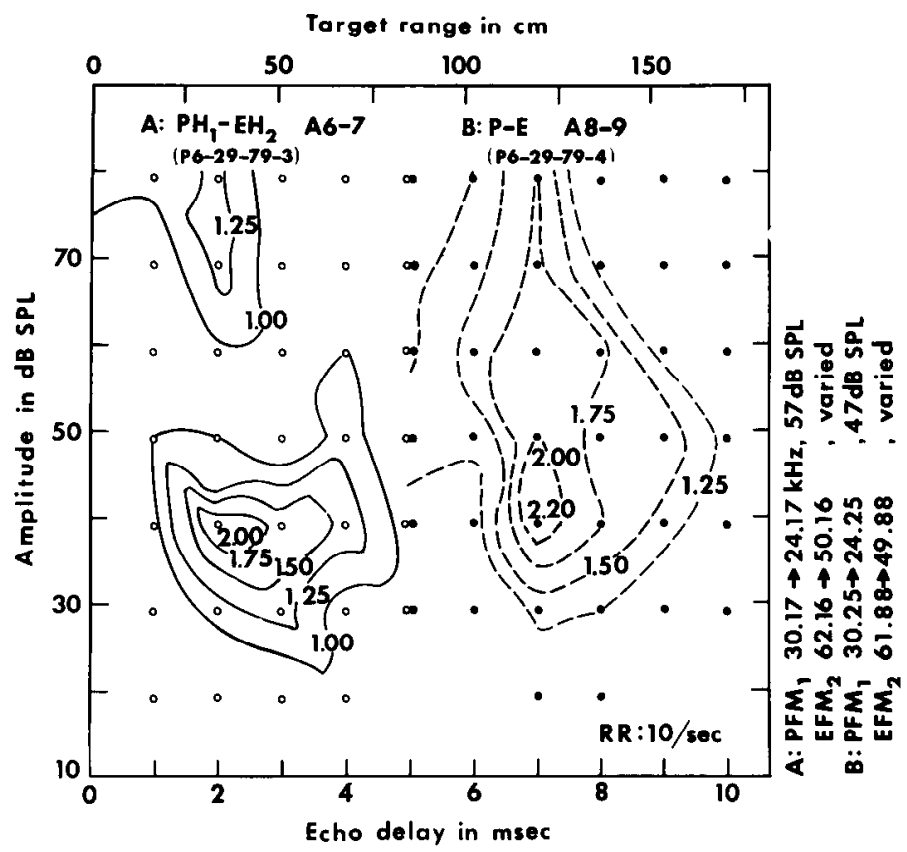

Figure 8. Isoimpulse count (isoresponse magnitude) contours of two delay-tuned neurons recorded from the same bat at 10/ sec. $A, \mathrm{FM}_{1}-\mathrm{FM}_{2}$ neuron with $\mathrm{BD}$ of $3.6 \mathrm{msec} ; B, \mathrm{FM}_{1}-\mathrm{FM}_{2}$ neuron with BD of 7 msec. Each open $(A)$ or closed $(B)$ circle represents the values of the echo delay and amplitude used for 100 presentations of pairs of stimuli at $10 / \mathrm{sec}$ in order to count the number of impulses per paired stimulus. Contour lines are drawn connecting echo delay versus echo amplitude coordinates eliciting equivalent numbers of impulses. The numbers associated with the contours indicate the average number of impulses per paired stimulus. For each neuron, the contours surround a tiny area of echo delay and amplitude values at which the respective neurons are excited maximally. The area of maximum response is near $2 \mathrm{msec}$ echo delay and $39 \mathrm{~dB}$ SPL amplitude for $A$ and 7 msec and $39 \mathrm{~dB}$ SPL for $B$. The contours with the lowest response magnitude (e.g., 1.00 in $A$ and 1.25 in $B$ ) correspond to the usual delay-tuning curves. Measurements were made 6 to $7 \mathrm{hr}(A)$ and 8 to $9 \mathrm{hr}(B)$ after administration of Innovar-vet. R.R., repetition rate of stimulation. 


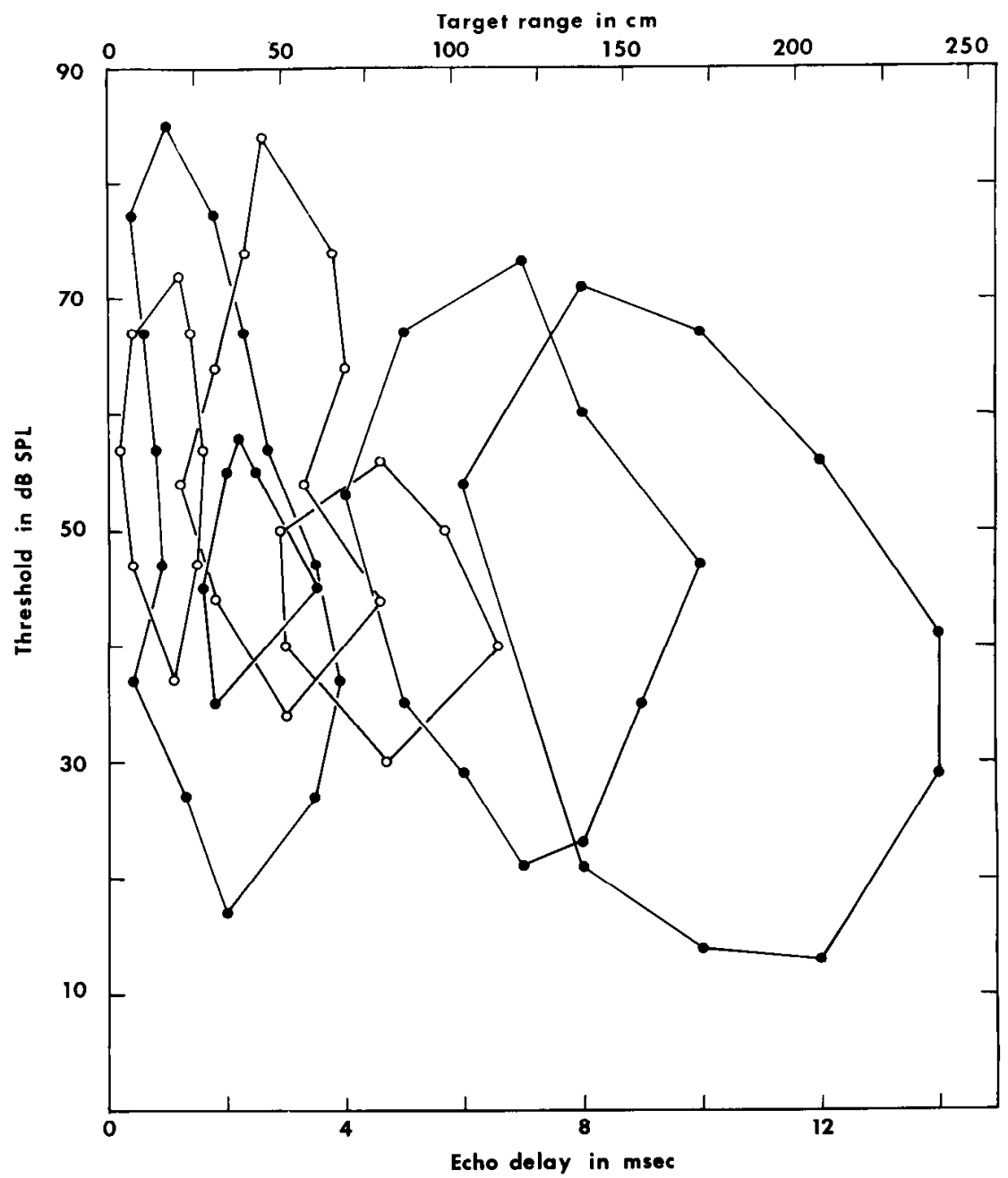

Figure 9. Delay-tuning curves of seven delay-tuned neurons measured at either $40 / \mathrm{sec}(O)$ or $100 / \mathrm{sec}(O)$ repetition rates. These curves were not all measured from a single animal but are simply drawn together here to demonstrate that populations of delay-tuned neurons can parcel out echo delays and, therefore, target ranges. The smaller the BD, the narrower are the delaytuning curves.

Each perpendicular penetration was characterized by neurons tuned to the same essential components in the pulse and echo and having nearly the same delay sensitivity. Figure $11 A$ illustrates the delay-tuning curves of four neurons encountered at $200-\mu \mathrm{m}$ intervals in a single perpendicular penetration. All were $\mathbf{F M}_{1}-\mathrm{FM}_{3}$ specialized. BDs measured at $10(a), 40(b)$, and $100(c) / \mathrm{sec}$ remained near $4 \mathrm{msec}$ for three of the four neurons. The exception was rather broadly delay tuned and it's BD was $2.5 \mathrm{msec}$ at 10 and $40 / \mathrm{sec}$. Tuning in time (i.e., the boundaries of the delay-tuning curves) was much more consistent from neuron-to-neuron than was the tuning for echo amplitude as seen by the greater variation in the upper thresholds. This variation of amplitude sensitivity with depth requires further study. The BDs of all of these neurons varied by $1 \mathrm{msec}$ or less at the three different repetition rates of stimulus pairs and thus met our operational definition for delay-tuned neurons.

Figure $11 B$ illustrates the BDs of neurons encountered in three different penetrations. In the penetration of the right graph (P12-4-79-P9), BDs recorded from the cell nearest the surface were nearly the same at all three repetition rates. All other neurons recorded at $200-\mu \mathrm{m}$ intervals throughout the depth of the cortex had BDs which differed depending on repetition rate by more than $1 \mathrm{msec}$; i.e., they are clearly all tracking neurons. As is typical for most perpendicular penetrations, at each recording site, the BDs at each repetition rate vary only slightly from the average value for the entire sample. The penetration in the center graph (P12-14-78-P7) containing tracking neurons with a less extensive $\mathrm{BD}$ shift also shows good agreement within the column for the $\mathrm{BD}$ values. The neurons recorded in another penetration (P12-4-78-P1 in the left graph) are all delay tuned, and except for the neuron recorded at $800 \mu \mathrm{m}$, they all were tuned to about $4 \mathrm{msec}$ echo delay. All such perpendicular penetrations indicated that $\mathrm{BD}$ is consistent throughout the cortical column at each repetition rate and that there are at least two types of columns representing either delay-tuned or tracking neurons.

Because delay-tuned columns are distinct from tracking columns and nearly all neurons within such columns have similar BDs and delay-tuning curves, we then could make tangential penetrations to study the distribution of delay-tuned columns in the plane of the cortex. According to our definition, BDs had to vary 1 msec or less to qualify a neuron as delay tuned. All others were classified as tracking neurons. 


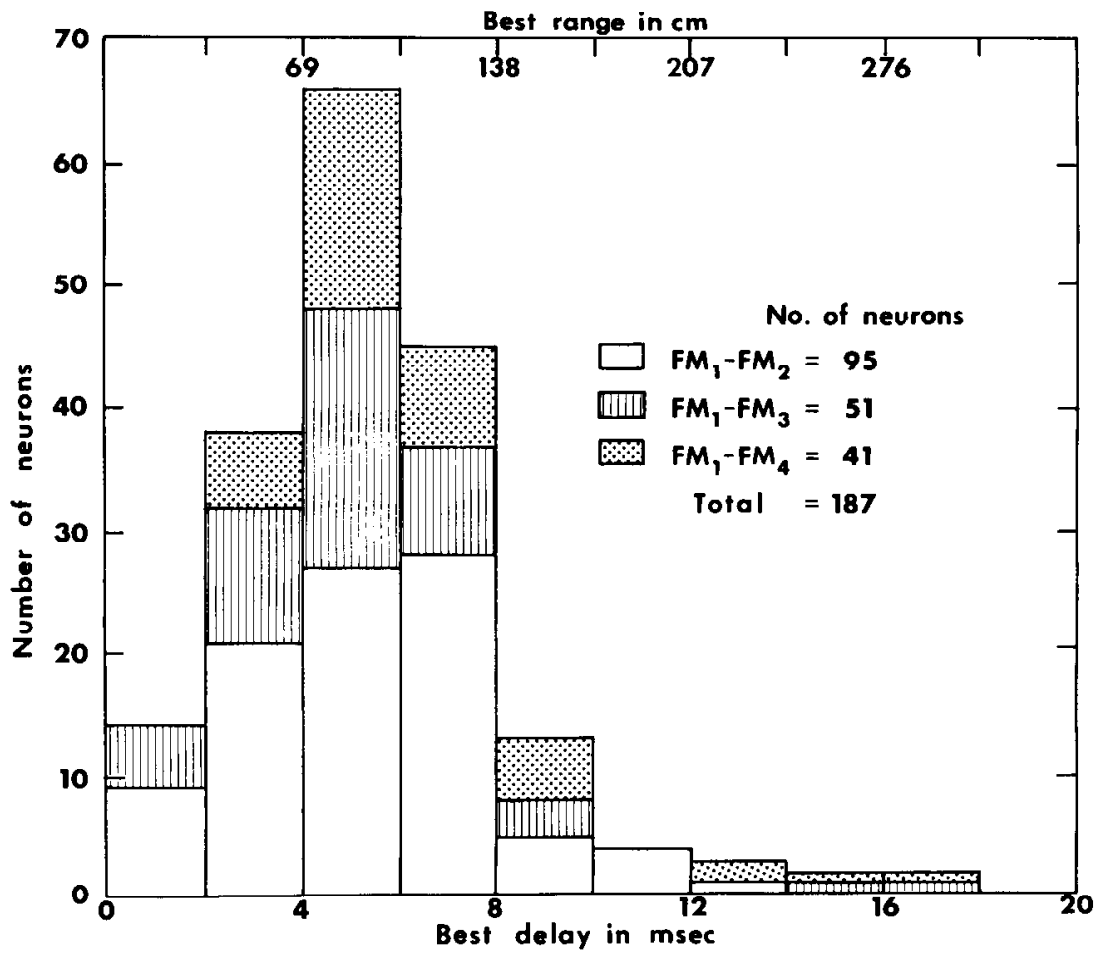

Figure 10. Distribution of BDs of 187 delay-tuned neurons with BDs of 0.4 to 18 msec. Ninety-five percent (95\%) of the sample have BDs less than $10 \mathrm{msec}$.

The data from one bat collected in one such study (from a total of six mapped hemispheres) are shown in Figure 12. The numbers indicate the BD (average value at three repetition rates) of delay-tuned neurons encountered beneath the place indicated by the solid circle on the drawing of cortical surface. $T$ indicates the location of tracking neurons.

First, it should be noted that the area contained at least three distinct clusters within which are found the three types of facilitation neurons: $\mathrm{FM}_{1}-\mathrm{FM}_{2}, \mathrm{FM}_{1}-\mathrm{FM}_{3}$, and $\mathrm{FM}_{1}-\mathrm{FM}_{4}$. In nearly all bats, these clusters were elongated rostrocaudally, as shown, although there were variations in their size and shape (Fig. 12B). The $\mathrm{FM}_{1}$ $\mathrm{FM}_{2}$ cluster was commonly the largest.

Within each cluster, BD increased systemically in the rostrocaudal direction as previously reported (Suga and O'Neill, 1979). BDs of the neurons sampled in the left hemisphere ( $L A C)$ shown in Figure 12 varied from 0.5 to $18 \mathrm{msec}$. Those neurons tuned to delays less than $4 \mathrm{msec}$ were actually found by making penetrations down into the bank of the temporal sulcus but are represented in an unfolded projection to the left of the shaded bar. Iso$B D$ contour lines drawn on the maps indicate a disproportionate amount of cortex occupied by neurons with BDs from 3 to $8 \mathrm{msec}$, in agreement with our pooled data already discussed (Fig. 10). Tracking neurons were rarely encountered, but in the right hemisphere $(R A C)$ in the example shown in Figure 12, one tangential electrode pass encountered three such cells in the dorsalmost part of the $\mathrm{FM}_{1}-\mathrm{FM}_{3}$ cluster.

In the rostrocaudal direction, we have shown previously a linear relationship between $\mathrm{BD}$ and the distance along the cortical surface for the range of echo delays between 1 and $8 \mathrm{msec}(16$ and $140 \mathrm{~cm})$. In the area containing longer $\mathrm{BDs}$, the $\mathrm{BD}$ increased more rapidly with cortical distance (Suga and O'Neill, 1979). Although the maps of BD were dissimilar for the left and right cortical hemispheres of individual bats, the FM-FM area was always identifiable in both.

\section{Discussion}

We have presented further evidence for the existence of a large area of auditory cortex which is functionally organized to represent time interval information (i.e., target range) by the location of the activity of delaytuned neurons. This FM-FM area contains no clear cochleotopic projection (Suga et al., 1981; N. Suga and W. E. O'Neill, manuscript in preparation). Rather, the neurons are grouped into three major clusters according to their selectivity for particular FM harmonics in the echo, each of which has its own rostrocaudal axis representing echo delay. Within a given cluster, neurons differ widely in their selectivity for the timing of acoustic events and only slightly in selectivity for frequency. They are unique among all auditory neurons so far described in their requirement for combinations of sounds with complex spectral and temporal relationships rather than simple frequency-amplitude combinations.

Two major classes of $\mathrm{FM}_{1}-\mathrm{FM}_{n}$ neurons are distinguishable on the basis of the degree of constancy of their best delays and delay-tuning curves. One type, which we sampled relatively rarely, has labile BDs and delay-tuning curves. The BDs shorten and curves become sharper as repetition rates of pulse-echo pairs increase, simulating conditions during target approach and interception. Because of this lack of constancy in the value of $\mathrm{BD}$ and the shape of the delay-tuning curve (Fig. 4), it is unlikely that these neurons play a role in encoding target range, 

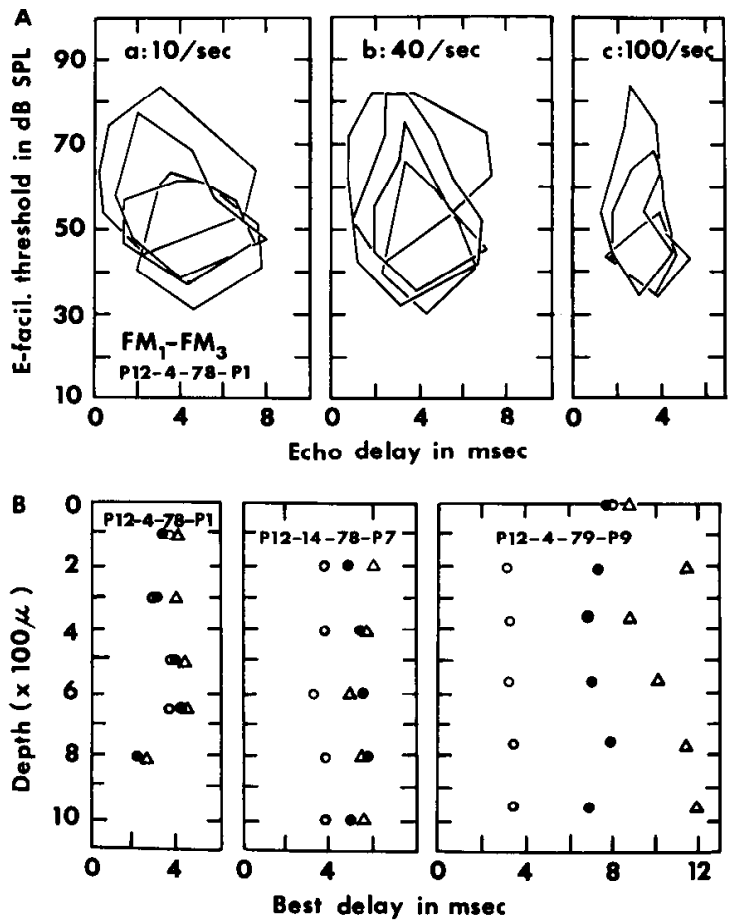

Figure 11. Delay tuning as a function of cortical depth. $A$, Delay-tuning curves of four $\mathrm{FM}_{1}-\mathrm{FM}_{3}$ neurons measured at 200$\mu \mathrm{m}$ intervals in a single perpendicular penetration at repetition rates of $10 / \mathrm{sec}(a), 40 / \mathrm{sec}(b)$, and $100 / \mathrm{sec}(c)$. BD remains nearly the same in $a, b$, and $c$, but there are some changes in the curves, especially in $c$. Bandwidths of the curves are relatively similar at each repetition rate, while the lower and upper thresholds vary. $B, \mathrm{BDs}$ measured at three repetition rates for neurons recorded in three different orthogonal electrode penetrations as a function of cortical depth. The penetration on the left is through a column of delay-tuned neurons. Penetrations depicted in the middle and right panels are through columns of tracking neurons. Note that, in each, BDs are generally the same for each neuron in the column. $\Delta, 10 / \mathrm{sec} ; 0,40 / \mathrm{sec} ; O$, $100 /$ sec.

although their response to echoes is clearly delay dependent. Their most interesting characteristic is the apparent ability to "track" an approaching target while simultaneously "rejecting" echoes from more distant targets which also are approaching but are not the object of interest; hence the tentative label, tracking neuron, with the caveat that the function implied by such a name requires further, more detailed study.

The necessity of such a response for the bat's successful orientation is not immediately obvious. At the very least, these neurons (and the delay-tuned neurons to be discussed), represent a selective "gating" or "attention" mechanism tied both to a specific motor activity (vocalization) and to the time course of incoming sensory information. Delay-sensitive neurons could focus the bat's "view" mainly on the target while simultaneously reducing the effect of potentially confusing echo images received from other objects.

The other type of delay-sensitive neuron, the delaytuned neurons, comprised the large majority of neurons that we encountered in the FM-FM area. They are distinguished by the constancy of both $\mathrm{BD}$ and delaytuning curves. Operationally, we included in this group only those delay-sensitive neurons whose BD varied 1 msec or less. The relative independence of these response properties from variations in pulse-echo repetition rate and duration dissociates them from such features of the bat's vocal behavior during target approach. They are, therefore, capable of encoding echo delay regardless of pulse rate and duration and, consequently, can be considered range tuned.

Although there are neurons whose BD and delay-tuning curve features are somewhat intermediate between the archetypical tracking and delay-tuned neuron, we, at present, feel justified in considering them to be two different neuronal classes on the basis of BD constancy alone. We made this decision first from the fact that delay-sensitive neurons with constant BDs (range tuned) also tended to have narrower delay-tuning curves whose shapes were independent of pulse-echo repetition rate. Secondly, in mapping the FM-FM area, we found clear rostrocaudal organization of the $\mathrm{BD}$ values of delay-

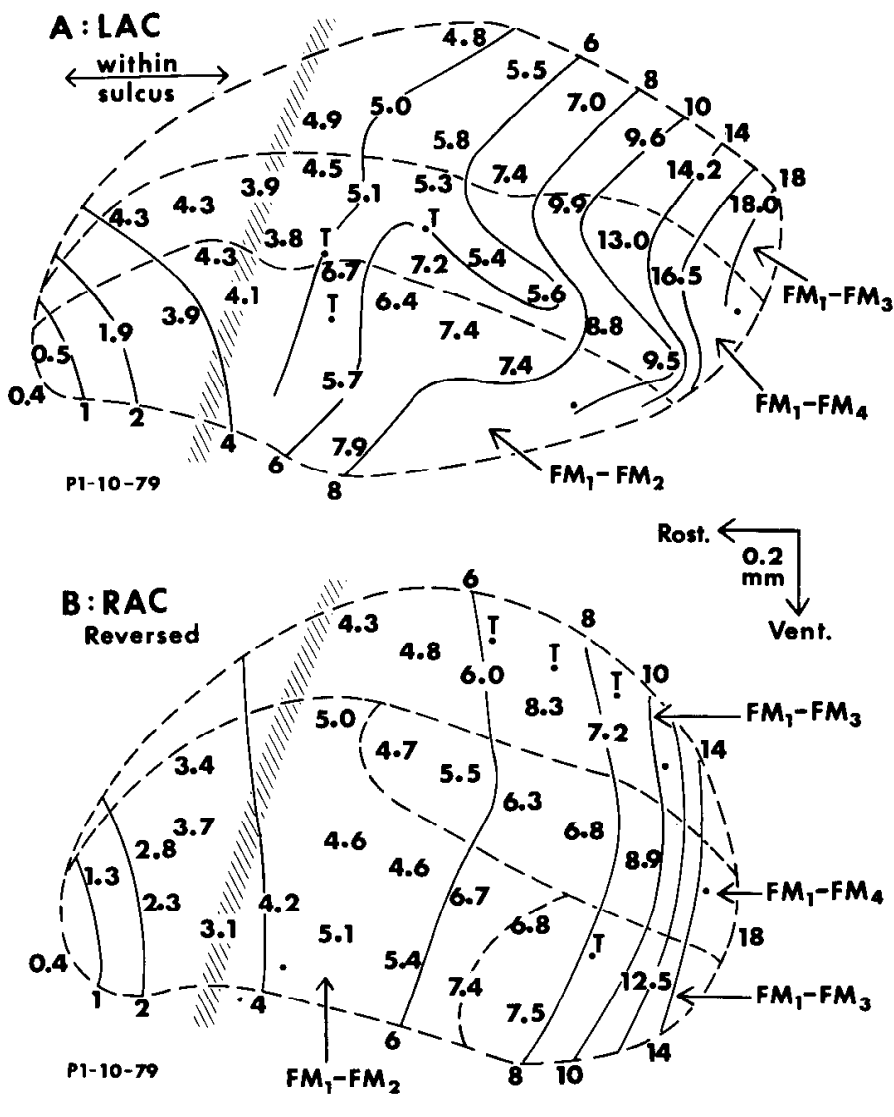

Bes $t$ delay in $m \sec (B R=B D \times 17.2 \mathrm{~cm})$

Figure 12. Distribution of BDs in the tangential plane of the FM-FM areas in both hemispheres of a single bat. The numbers refer to BDs measured at the locations indicated (solid circle) by multiple rostrocaudal tangential penetrations. Three or four clusters of neurons were found in the left ( $L A C)$ and right ( $R A C$, reversed) hemispheres, respectively, which contained exclusively $\mathrm{FM}_{1}-\mathrm{FM}_{3}, \mathrm{FM}_{1}-\mathrm{FM}_{4}$, or $\mathrm{FM}_{1}-\mathrm{FM}_{2}$ neurons. Iso-BD contours are drawn by interpolation between recording sites on the maps to indicate how $\mathrm{BD}$ increases in the rostrocaudal direction in each hemisphere and within each cluster. Samples taken to the left of the hatched bar were obtained by penetrations made into the sulcal fold. Note the disproportionately large area representing $\mathrm{BD}$ from 2 to $8 \mathrm{msec} . B R$, best range. 
tuned neurons, while tracking neurons were found rarely (their locations require further study).

These response property differences have quite different consequences for echo processing during target approach as well. Nearly all tracking neurons would be active regardless of the actual echo delay (target range) during the search and early approach stages. The Ushaped delay-tuning curves of tracking neurons are modified by changes in repetition rate to maintain nearly maximum sensitivity to echoes occurring during a broad time period between emissions of the FM components of successive biosonar signals. In contrast, range-tuned neurons are only able to respond to echoes when the target is at or very near a particular distance corresponding to the $\mathrm{BD}$. Thus, an individual range-tuned neuron would be activated only during a limited period of time during actual target pursuit. Feng et al. (1978) have found rangesensitive neurons in the nucleus intercollicularis (pericentral nucleus of the inferior colliculus) of the FM bat Eptesicus fuscus. Sullivan (1981) also has recently found range-sensitive neurons in the auditory cortex of the FM bat Myotis lucifugus. Therefore, target range information is processed eventually by range-sensitive neurons regardless of whether the biosonar signals employed are of the CF-FM or FM type.

Adaptations of $F M_{1}-F M_{n}$ neurons to range encoding. Many aspects of the response properties of $F M_{1}-F_{n}$ neurons illustrate adaptations for range processing. In regard to their frequency tuning, we have shown elsewhere (Suga et al., 1978) that the tuning curves for facilitation of these neurons are not sharp for either $\mathrm{PFM}_{1}$ or the EFM component. For both components, the essential FM sweeps may vary over a fairly broad range and still elicit facilitation. Thus, the decoding of the range information contained in pulse-echo time intervals is at least partly protected from spectral variations in both the emitted pulses and returning echoes. During Doppler shift compensation, target range processing may continue without interference from irrelevant changes in pulse or echo frequency.

The spectral selectivity for $\mathbf{F M}_{1}$ as the initial sound in a stimulus pair suggests that vocal self-stimulation by this component could act as a "primer" for subsequent response to an echo. A possible advantage for the use of the first harmonic component as a reference for measuring echo delays is the reduction of the possibility of jamming by the biosonar signals of nearby conspecifics. Sound spectrographs made in our laboratory indicate that the emitted $\mathrm{H}_{1}$ component is weak, usually 12 to 24 $\mathrm{dB}$ lower in amplitude than the predominant $\mathrm{H}_{2}$ component. Perhaps $\mathrm{H}_{1}$ is suppressed by vocal tract antiresonance mechanisms. It is possible that $\mathrm{H}_{1}$ is dissipated into the tissues and that the ears receive stimulation by $\mathrm{H}_{1}$ via bone conduction, while the pulses of other bats would reach the ears with insufficient $\mathrm{H}_{1}$ energy to stimulate the $\mathrm{FM}_{1}-\mathrm{FM}_{n}$ neurons. The ranging mechanism would thereby be relatively immune to conspecific jamming (Suga and O'Neill, 1979). Circumstantial support for this hypothesis can be derived from the lack of any delay-sensitive neurons responding to echo $\mathrm{FM}_{1}$ components, i.e., $\mathrm{FM}_{1}-\mathrm{FM}_{1}$ neurons. Detailed further studies of this problem are obviously necessary.

Another advantage that can be cited is related to the suppression of $\mathrm{H}_{1}$ in the emitted sounds and the detectability of the bat by tympanate moths. Fenton and Fullard (1979) showed that many tympanate moths hear best at 20 to $30 \mathrm{kHz}$ and that those species of bats emitting echolocation signals at higher frequencies than this have a theoretical advantage in approaching insect prey. By suppressing the $30-\mathrm{kHz}$ fundamental, the mustached bat may be able to approach these prey species much more closely before being detected.

Effects of nonessential components on the shape of the delay-tuning curve. One would predict, on the basis of traditional masking studies, that stimuli consisting only of essential components for facilitation would produce sharper delay-tuning curves than stimuli containing both essential and nonessential components. It was surprising to observe quite the contrary in our experiments on this issue (Fig. 7). Complete P-E pairs shrink both the dynamic range for echo amplitude and, to a lesser extent, the width of the delay-tuning curve. Total response magnitude of the neuron is normally reduced by the complete P-E stimulus, and this probably accounts for the increase in the unit's time and amplitude selectivity. The lowered overall evoked activity may, in fact, be the result of masking of their echo counterparts by the loud $\mathrm{H}_{2}$ or $\mathrm{H}_{3}$ components in the pulse. Doppler shifts in the echo and Doppler shift compensation would in any case minimize this masking in normal situations. This implies that our measurements of delay-tuning curves using only the essential pulse-echo components are conservative estimates of the true shape of the delay-tuning curve.

Target range resolution. Given that echo amplitude is within the response range of the neuron, the resolution of target range by individual range-tuned neurons depends on the temporal selectivity of the delay-tuning curve and the degree of change in response magnitude as a function of small changes of echo delay. By looking at delay-tuning curves of range-tuned neurons (e.g., Fig. 9), one can readily see that the selectivity decreases as a function of the BD. Temporal resolution by single neurons, therefore, is much better at close range. As a bat approaches a target, its estimate of target range should improve both because the echo is subjected to sharper delay-tuning curves and because the bat increases the number of range estimates per unit time by raising its pulse repetition rate. The behavioral data of Novick and Vaisnys (1964) on mustached bats indicate that the approach phase to small objects may involve as few as two or three pulse emissions. The necessity for rapid range estimation is most obvious in these conditions, and an array of range-tuned "filters" is ideally suited to the task.

Along the odotopic axis of the cortex, we found a disproportionately large number of neurons tuned to echo delays between 3 and $8 \mathrm{msec}$. In a previous paper (Suga and O'Neill, 1979), we calculated that the maximum resolution of distance at the single neuron level would be $2.0 \mathrm{~cm}$; this corresponds to an average $\mathrm{BD}$ increment of $0.06 \mathrm{msec}$ from one neuron to another in the rostrocaudal direction (interneuronal distance is about $20 \mu \mathrm{m}$ ). This assumes, of course, that range resolution is related to the increment in $\mathrm{BD}$ along the range axis. A calculated resolution of $2.0 \mathrm{~cm}$ correlates rather well with the older behavioral data obtained by Simmons (1971) for range discrimination in different species of long 
CF-FM and FM bats. It does not agree, however, with his more recent behavioral data (Simmons, 1979) from FM bats, which show range discrimination of only 0.1 $\mathrm{mm}$ (fully two orders of magnitude better than previous measurements of FM bats). We are unaware, at this time, of data from any level of the auditory system demonstrating neural mechanisms which may account for this new estimate.

Implications of the repetition of the odotopic axis in each $F M_{1}-F M_{n}$ cluster. Novick (1977), in discussing the possible function of harmonics in biosonar signals, suggested that they might allow bats to exploit a wider variety of prey sizes. The independent clusters of $\mathrm{FM}_{1}$ $\mathrm{FM}_{2}, \mathrm{FM}_{1}-\mathrm{FM}_{3}$, and $\mathrm{FM}_{1}-\mathrm{FM}_{4}$ neurons, each of which contains an odotopic axis, now provide us with neural evidence for such a possibility. In addition, signal theory suggests that FM signals with harmonics are capable of providing less ambiguous target range estimates. The mustached bat has, in effect, broadened the bandwidth of its signal by emitting multiple harmonics. The horseshoe bat, in contrast, emits long CF-FM signals in a single harmonic $\left(\mathrm{H}_{2}\right)$, with an FM component bandwidth of only 10 to $12 \mathrm{kHz}$. Simmons (1971) found that horseshoe bats do not discriminate range differences as well as bats using only broad band FM signals but that the performance was, in both cases, predicted by the FM bandwidth in the pulse. One wonders whether the mustached bat has overcome this apparent handicap of CFFM bats by exploiting the multiple harmonics and would thereby discriminate range better than the horseshoe bat.

The processing of temporal information in the auditory system. For most animals, including man, the temporal sequence of meaningful sounds is critical to their recognition. The apparent existence of phonetic "boundaries" for the perception of certain consonantvowel transitions in man (Liberman et al., 1967) and synthetic speech-like sounds in man (Miller et al., 1976) and in animals (Kuhl, 1976) has implied the operation of "feature detectors" acting as filters for certain temporal relationships in the spectra of such sounds. Recent evidence challenging the concept of strict laterality of speech processing in man is based on evidence that the processing of rapid changes in spectral features of speech sounds (e.g., frequency modulations or transitions) is carried out primarily in the left hemisphere, while processing of steady state sounds (e.g., vowels) shows no evidence of laterality (Schwartz and Tallal, 1980; Cutting, 1973).

The delay-sensitive neurons described here as well as those shown by Feng et al. (1978) and Suga et al. (1978) are the first examples of "temporal filters" existing at the single neuron level in the auditory system. In the mustached bat, these filters extract only the time interval information between 2 of the 16 spectral components $\left(\mathrm{PFM}_{1}\right.$ and $\mathrm{EFM}_{n}$ ) within a complex sound (P-E) for echo ranging. We have presented evidence elsewhere (Suga et al., 1979) that neurons in the CF/CF cortical area are sensitive to specific harmonic (but not temporal) relationships in complex sounds, and as such, they might be useful models for analyzing the formant or overtone content in complex sounds. Important time-dependent and overtone-dependent auditory perceptions may be subserved by just such neurons in other species, e.g., in the bullfrog Rana catesbeiana (Mudry and Capranica, 1978). We respectfully suggest that future work in higher auditory centers incorporate complex, biologically significant stimuli capable of exciting these types of cells.

The representation of acoustic space in the auditory cortex: An example of nontonotopic functional organization. Indirect evidence that tonotopic organization has functional significance is provided by the recent discovery of nontonotopically organized auditory areas in owls and bats. Knudsen and Konishi (1978) found that a portion of the auditory midbrain (MLD) of the barn owl (Tyto alba) is not tonotopically organized. Rather, neurons in this "space-mapped" region, by having spatially restricted receptive fields, are selective for the location of sounds around the owl's head (Konishi and Knudsen, 1978). These neurons are organized into an orderly map of acoustic space so that a sound's position is represented by the specific location of activity in the nucleus. Adjacent to this region is another subdivision of the MLD which is clearly tonotopically organized. More importantly, the space map is generated by neural interaction, not by the topography of the peripheral receptors.

The data that we have presented here demonstrate organization related to another cue for sound localization, namely range or depth perception. The neurons of the FM-FM area are so unique in their response properties that one cannot discuss their frequency selectivity in the usual terms. Pure tone stimuli are ineffective in driving these neurons, and no tonotopic axis is evident. The only clear organization discovered so far is the systematic change in delay tuning of neurons along the cortical surface. Here again, the brain represents, through a place mechanism, a map of auditory space. If these nontonotopic areas are functionally significant, then one may infer as a corollary that tonotopically organized areas also must have functional significance. The discovery of bands of aural dominance columns running perpendicular to isofrequency contours (Imig and Adrian, 1977; Middlebrooks et al., 1980) eventually may provide insight into the functional significance of tonotopic organization in cats. The significance of tonotopic organization in relation to cat (and monkey) behavior unfortunately remains obscure despite the many studies already published. However, the significance of tonotopic organization in relation to target movement analysis is now recognized in the mustached bat (Suga and Jen, 1976) and horseshoe bat (Ostwald, 1980).

The coexistence of areas organized according to best frequency adjacent to areas organized according to some other parameter in both the barn owl and mustached bat is a significant discovery in the auditory system. 'This suggests that there are at least two, and probably more, subdivisions of the auditory system specialized for processing different aspects of sound. The idea of parallel, semi-independent "transmission lines" subserving different "processors" decoding incoming information is not new, since modern fiber tracing techniques have shown many separate pathways beginning at the level of the cochlear nuclei and ramifying throughout the higher auditory system. Strong electrophysiological evidence also supports this view of parallel processing in the auditory pathway. In hindsight, it is not surprising that 
this functional segregation exists, since it would be unreasonable to suggest that an undifferentiated, tonotopically organized system is alone responsible for auditory perception because individual neurons do not appear able to decode even a few of the many different parameters associated with complex sounds. Rather, our evidence suggests that specific types of analyses are performed by discrete populations of neurons specialized for encoding only a small set of acoustic parameters. Our task, then, is to determine how different aspects of sound, which are individually analyzed in discrete regions of the brain, are integrated eventually into a perception of sound in space.

The behavioral specialization found in bats and owls has permitted correlation of the function of specific "processing areas" in their auditory systems with the animal's behavior. As a consequence, we propose that the traditional approach to the study of the auditory system be modified. Emphasis should be placed on the search for areas organized for the analysis of more complex, behaviorally relevant acoustic parameters rather than just for areas analyzing pure tones, clicks, or noise bursts. It seems quite unlikely that the auditory systems of these two species are unique, and it is perhaps not too bold at this time to predict that specialized processing areas will be found in the auditory systems of other species as well.

\section{References}

Cutting, J. E. (1973) A parallel between degree of encodedness and the ear advantage: Evidence from an ear monitoring task. J. Acoust. Soc. Am. 53: 368.

Feng, A. S., J. A. Simmons, and S. A. Kick (1978) Echo detection and target-ranging neurons in the auditory system of the bat, Eptesicus fuscus. Science 202: 645-648.

Fenton, M. B., and J. H. Fullard (1979) The influence of moth hearing on bat echolocation strategies. J. Comp. Physiol. 132: $77-86$.

Goldman, L. J., and O. W. Henson (1977) Prey recognition and selection by the constant frequency bat, Pteronotus $p$. parnellii. Behav. Ecol. Sociobiol. 2: 411-419.

Imig, T. J., and H. O. Adrian (1977) Binaural columns in the primary field (A1) of cat auditory cortex. Brain Res. 138: 241-257.

Knudsen, E. I., and M. Konishi (1978) Space and frequency are represented separately in the auditory midbrain of the owl. J. Neurophysiol. 41: 870-884.

Konishi, M., and E. I. Knudsen (1978) Center-surround organization of auditory receptive fields in the owl. Science 202 . 778-780.

Kuhl, P. (1976) Speech perception by the chinchilla: Categorical perception of synthetic alveolar plosive consonants. J. Acoust. Soc. Am. 60: 5581 Abstr.

Liberman, A. M., F. S. Cooper, D. P. Shankweiler, and M. Studdert-Kennedy (1967) Perception of the speech code. Psychol. Rev. 74: 431-461.

Middlebrooks, J. C., R. W. Dykes, and M. M. Merzenich (1980) Binaural response-specific hands in primary auditory cortex (AI) of the cat. Topographical organization orthogonal to isofrequency contours. Brain Res. 181: 31-48.

Miller, J., C. Weir, R. Pastore, W. Kelly, and R. Dooling (1976) Discrimination and labelling of noise-buzz sequences with varying noise-lead times: An example of categorical perception. J. Acoust. Soc. Am. 60: 410-417.

Mudry, K. M., and R. R. Capranica (1978) Electrophysiological evidence for auditory responsive areas in the diencephalon and telencephalon of the bullfrog, Rana catesbeiana. Soc. Neurosci. Abstr. 4: 101.
Novick, A. (1977) Acoustic orientation. In Biology of Bats, W. A. Wimsatt, ed., Vol. III, pp. 74-289, Academic Press, New York.

Novick, A., and R. Vaisnys (1964) Echolocation of flying insects by the bat, Chilonycteris parnellii. Biol. Bull. 128: 297-314.

O'Neill, W. E., and N. Suga (1979) Target range-sensitive neurons in the auditory cortex of the mustached bat. Science 203: 69-73.

Ostwald, J. (1980) The functional organization of the auditory cortex in the CF-FM bat, Rhinolophus ferrumequinum. In Animal Sonar Systems, R. -G. Busnel and J. F. Fish, eds., pp. 953-956, Plenum, New York.

Pollak, G. D. (1980) Organizational and encoding features of single neurons in the inferior colliculus of bats. In Animal Sonar Systems, R. -G. Busnel and J. F. Fish, eds., pp. 549587, Plenum, New York.

Schnitzler, H. -U. (1970) Echoortung bei der Fledermaus Chilonycteris rubiginosa. Z. Vegl. Physiol. 68: 25-38.

Schnitzler, H. -U., and O. W. Henson (1980) Performance of airborne animal sonar systems. I. Microchiroptera. In Animal Sonar Systems, R. -G. Busnel and J. F. Fish, eds., pp. 109 182, Plenum, New York.

Schuller, G. (1974) The role of overlap of echo with outgoing orientation sound in the bat Rhinolophus ferrumequinum. Naturwissenschaften 4: 171-172.

Schuller, G., and N. Suga (1976) Storage of Doppler-shift information in the echolocation system of the "CF-FM" bat Rhinolophus ferrumequinum. J. Comp. Physiol. 105: 9-14.

Schwartz, J., and P. Tallal (1980) Rate of acoustic change may underlie hemispheric specialization for speech perception. Science 207: 1380-1381.

Simmons, J. A. (1971) The sonar receiver of the bat. Ann. N. Y. Acad. Sci. 188: 161-174.

Simmons, J. A. (1973) The resolution of target range by echolocating bats. J. Acoust. Soc. Am. 54: 157-173.

Simmons, J. A. (1979) Perception of echo phase information in bat sonar. Science 204: 1336-1338.

Smith, J. D. (1972) Systematics of the Chiropteran Family Mormoopidae, miscellaneous publication, University of Kansas, Museum of Natural History, No. 56, Lawrence, KS.

Suga, N., and P. H. -S. Jen (1976) Disproportionate tonotopic representation for processing CF-FM sonar signals in the mustached bat auditory cortex. Science 194: 542-544.

Suga, N., and P. H. -S. Jen (1977) Further studies on the peripheral auditory system of CF-FM bats specialized for fine frequency analysis of Doppler-shifted echoes. J. Exp. Biol. 69: 207-232.

Suga, N., and T. Manabe (1982) Neural basis of amplitudespectrum representation in the auditory cortex of the mustached bat. J. Neurophysiol., in press.

Suga, N., and W. E. O'Neill (1979) Neural axis representing target range in the auditory cortex of the mustached bat. Science 206: 351-353.

Suga, N., W. E. O'Neill, and T. Manabe (1978) Cortical neurons sensitive to combinations of information-bearing elements of biosonar signals in the mustache bat. Science 200: 778-781.

Suga, N., W. E. O'Neill, and T. Manabe (1979) Harmonicsensitive neurons in the auditory cortex of the mustached bat. Science 203: 270-274.

Suga, N., K. Kuzirai, and W. E. O'Neill (1981) How biosonar information is represented in the bat cerebrum. In Neuronal Mechanisms in Hearing, J. Syka, ed., pp. 197-220, Plenum, New York.

Sullivan, W. E., III (1981) Cortical representation of target distance information in the auditory system of the echolocating bat, Myotis lucifugus. Doctoral dissertation, Neuroscience Program, Washington University, St. Louis, MO.

von Békésy, G. (1960) Experiments in Hearing, McGraw-Hill, New York. 